\title{
A Lagrangian Nodal Integration Method for Free-Surface Fluid Flows
}

\author{
Alessandro Franci ${ }^{\mathrm{a}, *}$, Massimiliano Cremonesi $^{\mathrm{b}}$, Umberto Perego $^{\mathrm{b}}$, Eugenio Oñate $^{\mathrm{a}}$ \\ anternational Center for Numerical Methods in Engineering (CIMNE), Barcelona, Spain \\ ${ }^{b}$ Department of Civil and Environmental Engineering, Politecnico di Milano, Piazza Leonardo da Vinci 32 , \\ Milano, Italy
}

\begin{abstract}
We present a Lagrangian nodal integration method for the simulation of Newtonian and nonNewtonian free-surface fluid flows. The proposed nodal Lagrangian method uses a finite element mesh to discretize the computational domain and to define the (linear) shape functions for the unknown nodal variables, as in the standard Particle Finite Element Method (PFEM). In this approach, however, the integrals are performed over nodal patches and not over elements, and strains/stresses are defined at nodes and not at Gauss points. This allows to limit the drawbacks associated with the remeshing and leads to a more accurate stress computation than in the classical elemental PFEM. Several numerical tests, in 2D and in 3D, are presented to validate the proposed nodal PFEM. In all cases, the method has shown a very good agreement with analytical solutions and with experimental and numerical results from the literature. A thorough comparison between nodal and elemental PFEMs is also presented, focusing on crucial issues, such as solution accuracy, convergence, mass conservation and sensitivity to mesh distortion.
\end{abstract}

Keywords: Nodal Integration, PFEM, Free-Surface, Nodal-PFEM

\section{Introduction}

In this work, we present, analyze and validate a nodal integration scheme in the framework of the Particle Finite Element Method (PFEM) for the numerical simulation of free-surface fluids.

\footnotetext{
${ }^{\star}$ Fully documented templates are available in the elsarticle package on CTAN

* Corresponding author

Email addresses: falessandro@cimne.upc.edu (Alessandro Franci ), umberto.perego@polimi.it (Umberto Perego), onate@cimne.upc.edu (Eugenio Oñate)

URL: massimiliano.cremonesi@polimi.it (Massimiliano Cremonesi)
} 
The PFEM has shown to be an efficient method for the simulation of large displacement problems, such as free-surface fluid dynamics flows [17, 33, 38, geotechnical applications [35, 36], fluidstructure interaction [34, 31] and melting [37, 30] problems, among others.

In the standard PFEM [15], the governing equations are integrated over the mesh elements in the classical Finite Element Method (FEM) fashion, and the mesh nodes are moved according to the solution of the governing equations, in a purely Lagrangian way. This allows tracking accurately the evolving shape of the computational domains, also in the case of large deformation processes. However, to maintain a good quality of the FEM mesh, it is required to rebuild the discretization whenever it becomes excessively distorted.

At each PFEM remeshing step, the elements of the mesh are disgregated and a new triangulation is built over the cloud points formed by the nodes of the previous mesh. The loss of the elemental information makes the PFEM more suitable for problems that do not require the storage of historical variables at the element level. A proof of this is the reduced literature of PFEM formulations for solid mechanics, versus the extended one on fluid-type applications (e.g. see [25] for a recent review of the PFEM literature).

In this scenario, nodal integration methods, computing and storing stress and material history variables directly at the mesh nodes, are expected to be less affected by remeshing operations than an elemental approach and, globally, to adapt more naturally to the PFEM solution scheme.

However, only very recently the use of nodal integration in a PFEM framework has been investigated and successfully applied to geotechnical problems [9, 10. In these works, the authors proposed the so-called Smoothed Particle Finite Element Method (SPFEM), inspired by the well-established Smoothed Finite Element Method (SFEM) [5, 7, 2, 3,

Besides the already referred works on nodal integration applied to PFEM, one of the first attempts to use nodal integration combined with the FEM was in [47, where a node-based formulation was applied to the volumetric component of the strain energy while treating the deviatoric component as in the standard elements. In [11, the same idea has been extended by applying the nodal averaging process to the whole strain tensor, but only for small strain elasticity, while [12] applied the nodal averaging approach also to the large strain regime. These works constitute the first attempts to apply nodal integration within a FEM framework in solid dynamics. More recently, [40] proposed a stabilized nodal integration scheme for linear tetrahedral elements to avoid spurious low-energy modes showing good performances for plasticity, nearly incompressible materials and 
acute bending. 44 generalized the aforementioned technique and showed its application to linear elastic small-deformation models. The same formulation has been extended to plates in [45] and also to elastoplastic problems [46]. As shown in [44] there is a clear connection between the stabilized conforming nodal integration and the integration technique used in various meshfree methods (e.g. see [48, 49]).

The use of nodal integration methods in large displacement processes is attractive not only because remapping of the state variables (and its associated interpolation error) is avoided [39, but also because, node-based FEM models are expected to be less sensitive to the (bad) quality of the discretization [8]. This feature is crucial in a PFEM framework, as it allows reducing the number of remeshing events and their associated drawbacks: computational cost, arbitrary volume variation, and equilibrium perturbation.

Furthermore, as stated in [41], the stresses of mixed FEM with node-based finite element spaces for strains/stresses can be, at least in principle, second-order accurate. On the other hand, due to the larger number of supporting elements for node-based smoothing domains, nodal integration methods lead inevitably to stiffness matrices with a larger bandwidth versus the elemental counterparts, and thus to a higher computational cost for the linear system solution [1].

All these features explain the interest in exploring and analyzing a nodal integration solution scheme in the PFEM framework. Our aim in this work is to analyze the possibility of a nodal PFEM formulation in the context of free-surface fluid dynamics problems. The results of this study, besides representing a novelty in the literature, will also provide necessary information for the possible extension of this formulation to a unified nodal PFEM for fluid and solid problems with phase change.

The proposed formulation is obtained by redefining the stabilized mixed velocity-pressure formulation used in 24] with the nodal integration scheme proposed in the aforementioned references for solid mechanics (e.g. [11]). Indeed, the integration is here performed over the nodal areas (or nodal volumes in 3D), and not over the contours of the nodal patch. Note that the resulting integration method is the same as SFEM [1] and SPFEM [10, when linear triangular or tetrahedral elements are employed. Following [24, the Finite Increment Calculus (FIC) is used to stabilize the formulation, allowing us to make more accurate and focused comparisons between the nodal and the elemental schemes.

The proposed nodal method will be tested with both Newtonian and non-Newtonian fluids, 
in $2 \mathrm{D}$ and $3 \mathrm{D}$. The numerical formulation will be validated against laboratory tests, analytical solutions, and other numerical formulations.

The structure of the paper is the following. In Section 2 the governing equations of the problem are presented. In Section 3 , the weak form and the FEM approximation are introduced. In Section 4. the fully discretized form of the problem is derived according to the proposed nodal integration scheme. A brief mention of the used stabilization is presented in Section 5 . In Section 6 the linearized form of the stabilized problem is presented, while the solution scheme is described for a general time step in Section 7. Section 8 presents the validation tests for the proposed nodal PFEM formulation. Finally, in Section 9 the main outputs of this work are recalled.

\section{Governing equations}

In this work, we model the dynamics of incompressible Newtonian and non-Newtonian fluids with free-surface. The governing equations are the balance of linear momentum and the mass conservation (Navier-Stokes equations). As in the standard PFEM [15], an Updated Lagrangian formulation is here considered. In this framework, the linear momentum equations read

$$
\rho \dot{\boldsymbol{v}}-\nabla \cdot \boldsymbol{\sigma}-\boldsymbol{b}=\mathbf{0} \quad \text { in } \Omega \times(0, T)
$$

where $\rho$ is the fluid density, $\dot{\boldsymbol{v}}$ is the time variation of the velocity component, $\boldsymbol{\sigma}$ is the Cauchy stress tensor, $\boldsymbol{b}$ are the body forces per unit of volume, $\Omega$ is the updated/deformed fluid domain, and $(0, T)$ the time interval.

To deal with the fluid incompressibility, the Cauchy stress tensor is decomposed into its deviatoric and volumetric components as

$$
\boldsymbol{\sigma}=\boldsymbol{\sigma}^{\prime}+p \mathbf{I}
$$

where $\sigma^{\prime}$ is the deviatoric part of the Cauchy stress tensor, $p$ is the pressure (positive in a tensile state) and $\mathbf{I}$ is the second order identity tensor. Furthermore, the deviatoric stress can be related to the deviatoric strain rate $\boldsymbol{d}^{\prime}$ through:

$$
\sigma^{\prime}=2 \tilde{\mu} d^{\prime}
$$

where $\boldsymbol{d}^{\prime}$ is defined as:

$$
\boldsymbol{d}^{\prime}=\boldsymbol{d}-\frac{d_{v}}{3} \mathbf{I}
$$


with

$$
\boldsymbol{d}(\boldsymbol{v})=\frac{1}{2}\left(\nabla \boldsymbol{v}+[\nabla \boldsymbol{v}]^{T}\right) \quad d_{v}(\boldsymbol{v})=\nabla \cdot \boldsymbol{v}=\operatorname{trace}(\boldsymbol{d})
$$

$\tilde{\mu}$ is the real viscosity for Newtonian fluid, while for non-Newtonian fluids represents an apparent viscosity [42].

Considering a weakly compressible framework [19, 20], the mass conservation equation can be written as:

$$
\frac{1}{\kappa} \dot{p}-\nabla \cdot \boldsymbol{v}=\mathbf{0} \quad \text { in } \Omega \times(0, T)
$$

where $\kappa$ is the bulk modulus of the fluid. Note that the standard divergence-free form of the continuity equation $(\nabla \cdot \boldsymbol{v}=0)$ is recovered for the bulk modulus going to infinity.

The system of Eqs.(1-6) must be supplemented with appropriate boundary conditions on the Dirichlet $\left(\Gamma_{v}\right)$ and Neumann $\left(\Gamma_{t}\right)$ boundaries, which respectively read:

$$
\begin{array}{ll}
\boldsymbol{v}-\hat{\boldsymbol{v}}=0 & \text { on } \Gamma_{v} \\
\boldsymbol{\sigma} \cdot \boldsymbol{n}-\hat{\boldsymbol{t}}=0 & \text { on } \Gamma_{t}
\end{array}
$$

where $\hat{\boldsymbol{v}}$ and $\hat{\boldsymbol{t}}$ are the prescribed velocities and tractions, respectively, and $\boldsymbol{n}$ is the outgoing normal vector to fluid boundaries.

\section{Weak form and FEM approximation}

The weighted residual form of the momentum equations is obtained by integrating Eq.(1) over the computational domain $\Omega$, and by multiplying its terms by a vector test functions $(\boldsymbol{w})$. After integrating by parts the term involving the stress $\boldsymbol{\sigma}$ and introducing the Neumann boundary conditions (Eq. (8)), the weak form of momentum equations reads:

$$
\int_{\Omega} \rho \boldsymbol{w} \cdot \dot{\boldsymbol{v}} d \Omega+\int_{\Omega} \boldsymbol{d}(\boldsymbol{w}): \boldsymbol{\sigma} d \Omega-\int_{\Omega} \boldsymbol{w} \cdot \boldsymbol{b} d \Omega-\int_{\Gamma_{t}} \boldsymbol{w} \cdot \hat{\boldsymbol{t}} d \Gamma=\mathbf{0}
$$

Analogously, the weak form of the continuity equation is obtained by multiplying Eq.(6) by a scalar test function $(q)$ for the pressure:

$$
\int_{\Omega} \frac{1}{\kappa} q \dot{p} d \Omega-\int_{\Omega} q \nabla \cdot \boldsymbol{v} d \Omega=0
$$


As in the standard FEM, the domain $\Omega$ is discretized over a standard finite elements mesh. Three-noded triangles and four-noded tetrahedra are employed for 2D and 3D problems, respectively. For simplicity, in the following, we focus on the $2 \mathrm{D}$ case only. The extension to $3 \mathrm{D}$ is straightforward.

Following a standard Galerkin approach, the velocities, the pressure and the test functions are interpolated with linear shape functions $N$ :

$$
\begin{array}{ll}
\boldsymbol{v}(x, t)=\boldsymbol{N}(x) \overline{\boldsymbol{v}}(t) & \boldsymbol{w}(x, t)=\boldsymbol{N}(x) \overline{\boldsymbol{w}}(t) \\
p(x, t)=\boldsymbol{N}(x) \overline{\boldsymbol{p}}(t) & q(x, t)=\boldsymbol{N}(x) \overline{\boldsymbol{q}}(t)
\end{array}
$$

where the upper symbol $(\bar{\cdot})$ denotes a vector containing the nodal variables. Consequently, substituting Eqs. (11) into Eq.(9), the following weak form of the linear momentum equations is obtained:

$$
\int_{\Omega} \rho \boldsymbol{N}^{T} \boldsymbol{N} d \Omega \dot{\overline{\boldsymbol{v}}}+\int_{\Omega} \boldsymbol{B}^{T} \boldsymbol{\sigma} d \Omega-\int_{\Omega} \boldsymbol{N}^{T} \boldsymbol{b} d \Omega-\int_{\Gamma_{t}} \boldsymbol{N}^{T} \hat{\boldsymbol{t}} d \Gamma=\mathbf{0}
$$

where the operator $\boldsymbol{B}$ is defined such that [43]:

$$
\boldsymbol{d}(\boldsymbol{x}, t)=\boldsymbol{B}(\boldsymbol{x}) \overline{\boldsymbol{v}}(t)
$$

The discrete form of the continuity equation is obtained analogously. Eq. (11) is introduced into Eq. 10p leading to:

$$
\int_{\Omega} \frac{1}{\kappa} \boldsymbol{N}^{T} \boldsymbol{N} d \Omega \dot{\bar{p}}-\int_{\Omega} \boldsymbol{N}^{T} d_{v} d \Omega=\mathbf{0}
$$

where the volumetric deformation rate $d_{v}$ is computed from the discrete divergence operator $\tilde{\boldsymbol{B}}[24]$ as:

$$
d_{v}(\boldsymbol{x}, t)=\tilde{\boldsymbol{B}}(\boldsymbol{x}) \overline{\boldsymbol{v}}(t)
$$

\section{Nodal integration}

Contrary to an elemental approach, the numerical integration of the weak form (Eqs.(12,14)) is not performed over the elemental areas $\Omega^{\alpha}$, but over nodal patches of the triangular elements, whose area $\Omega^{I}$ is defined as

$$
\Omega^{I}=\sum_{\alpha=1}^{n e^{I}} \frac{\Omega^{\alpha}}{3}
$$


where $n e^{I}$ is the number of neighbor elements of node $I$ (i.e. elements sharing node $I$ ), such that

$$
\int_{\Omega} d \Omega=\sum_{I=1}^{N N} \Omega^{I}=\sum_{\alpha=1}^{N E} \Omega^{\alpha}
$$

where $N N$ and $N E$ are the total number of nodes and elements of the mesh, respectively 1 . Figure 1 shows a graphical representation of the nodal patch of a generic node $I$.

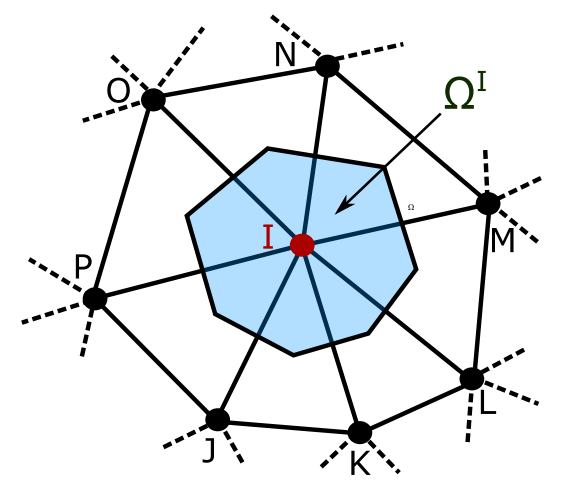

Figure 1: Nodal area $\Omega^{I}$ for node $I$.

The integral of a generic function $\boldsymbol{f}(\boldsymbol{x})$ over the whole domain $\Omega$ is computed nodally as follows:

$$
\int_{\Omega} \boldsymbol{f}(\boldsymbol{x}) d \Omega=\sum_{I=1}^{N N} \int_{\Omega^{I}} \boldsymbol{f}(\boldsymbol{x}) d \Omega
$$

and the integrals over the nodal patches are approximated as [39]:

$$
\sum_{I=1}^{N N} \int_{\Omega^{I}} \boldsymbol{f}(\boldsymbol{x}) d \Omega \cong \sum_{I=1}^{N N} \boldsymbol{f}\left(\boldsymbol{x}^{\boldsymbol{I}}\right) \Omega^{I}
$$

Remark 1. In the definition of nodal area (nodal volume in 3D), it is required neither the definition of nodal patch boundaries nor the computation of their normals. The unique requirement that must be satisfied is that the elemental surface has to be equally subdivided by the shared nodes. In other words, it must be ensured that each triangular element must contribute with a third of its area to the nodal patch of each one of its shared nodes. In this sense, the definition of the nodal area at the boundaries of the computational domain does not imply any further algebraic or geometrical complexities.

\footnotetext{
${ }^{1}$ Superscripts with small greek letters denote an element, whereas those with capital italic letters refer to a node.
} 


\subsection{Fully discretized form}

The integration procedure described in the previous section is here applied to Eqs. 12 . 14 to derive the fully discretized form of the original problem. For the sake of clarity, the linear momentum and the continuity equations are treated separately.

\subsubsection{Linear momentum equations}

Exploiting the partition of the domain in patches (see Eq. (18), the linear momentum equations (Eq. 12) are rewritten as

$$
\sum_{I=1}^{N N} \int_{\Omega^{I}} \rho N^{I} N^{I} d \Omega \dot{\overline{\boldsymbol{v}}}^{I}+\sum_{I=1}^{N N} \int_{\Omega^{I}}\left[\overline{\boldsymbol{B}}^{I}\right]^{T} \boldsymbol{\sigma} d \Omega-\sum_{I=1}^{N N} \int_{\Omega^{I}} N^{I} \boldsymbol{b} d \Omega-\sum_{I=1}^{N N} \int_{\Gamma_{t}^{I}} N^{I} \hat{\boldsymbol{t}} d \Gamma=\mathbf{0}
$$

where the sum should be interpreted as a standard FEM assembly operator. The nodal operator $\overline{\boldsymbol{B}}$ and the nodal stresses $\overline{\boldsymbol{\sigma}}$ will be defined in Section 4.2

Finally, using Eq. 19 and being the linear shape function equal to one at the nodes, the following nodal integrated form of the linear momentum is obtained

$$
\underbrace{\sum_{I=1}^{N N} \rho \Omega^{I} \dot{\boldsymbol{v}}^{I}}_{\boldsymbol{F}^{\boldsymbol{d}}}+\underbrace{\sum_{I=1}^{N N}\left[\overline{\boldsymbol{B}}^{I}\right]^{T} \overline{\boldsymbol{\sigma}}^{I} \Omega^{I}}_{\boldsymbol{F}^{\boldsymbol{\sigma}}}-\underbrace{\sum_{I=1}^{N N} \boldsymbol{b}^{I} \Omega^{I}-\sum_{I=1}^{N N} \alpha_{\Gamma}^{I} \hat{\boldsymbol{t}}^{I} \Gamma^{I}}_{\boldsymbol{F}^{\boldsymbol{e}}}=\mathbf{0}
$$

where $\alpha_{\Gamma}^{I}$ is equal to one if the node $I$ belongs to a Neumann boundary and equal to zero otherwise, $\Gamma^{I}$ is the nodal contour length (area, in 3D), and $\boldsymbol{F}^{\boldsymbol{d}}, \boldsymbol{F}^{\boldsymbol{\sigma}}$, and $\boldsymbol{F}^{\boldsymbol{e}}$ are the dynamic, internal and external equivalent nodal forces vector, respectively.

Remark 2. Note that the mass matrix in Eq.21, multiplying nodal accelerations, is diagonal by construction.

To obtain the fully discretized form of the linear momentum equations, the nodal accelerations $\dot{\overline{\boldsymbol{v}}}$ must be defined. Following [24], a second order Newmark scheme is used. Hence, for a time step interval $\left[{ }^{n} t ;{ }^{n+1} t\right]$ of length $\Delta t$, acceleration is computed as:

$$
{ }^{n+1} \dot{\overline{\boldsymbol{v}}}=\frac{2}{\Delta t}\left({ }^{n+1} \overline{\boldsymbol{v}}-{ }^{n} \overline{\boldsymbol{v}}\right)-{ }^{n} \dot{\overline{\boldsymbol{v}}}
$$

For convenience, Eq. 21] is rewritten in residual form as:

$$
R_{m}:=F^{d}+F^{\sigma}-F^{e}=0
$$




\subsubsection{Continuity equation}

Following the steps used to derive the fully discretized momentum balance, the fully discretized residual form of the continuity equations (Eq.(14) is also obtained. Using Eqs.(5 18), Eq.(14) is rewritten as

$$
\sum_{I=1}^{N N} \int_{\Omega^{I}} \frac{1}{\kappa} N^{I} N^{I} d \Omega \dot{\bar{p}}^{I}-\sum_{I=1}^{N N} \int_{\Omega^{I}} N^{I} \bar{d}_{v}^{I} d \Omega=0
$$

where the nodal volumetric deformation rate $\bar{d}_{v}^{I}$ will be defined in Section 4.2 together with the other nodal stress/strain measures.

Integrating numerically over the mesh nodes using Eq. $(19)$ and being the shape functions equal to one at the nodes, Eq. 224) simplifies to

$$
\underbrace{\sum_{I=1}^{N N} \frac{1}{\kappa} \Omega^{I} \dot{\bar{p}}^{I}}_{\boldsymbol{F}^{\boldsymbol{p}}}-\underbrace{\sum_{I=1}^{N N} \bar{d}_{v}^{I} \Omega^{I}}_{\boldsymbol{F}^{\boldsymbol{v}}}=0
$$

Following [24, the pressure time variation is computed for a time step interval $\left[{ }^{n} t ;{ }^{n+1} t\right]$ of duration $\Delta t$ as

$$
{ }^{n+1} \dot{\bar{p}}=\frac{1}{\Delta t}\left({ }^{n+1} \bar{p}-{ }^{n} \bar{p}\right)
$$

For convenience, Eq. 25$]$ is rewritten in residual form as:

$$
\boldsymbol{R}_{c}:=\boldsymbol{F}^{p}-\boldsymbol{F}^{v}=\mathbf{0}
$$

where $\boldsymbol{F}^{p}$ and $\boldsymbol{F}^{\boldsymbol{v}}$ have been defined in Eq.25.

\subsection{Nodal strains and stresses}

In this nodal approach, the nodal stresses $\overline{\boldsymbol{\sigma}}$ are computed using Eqs.(2, 5 as

$$
\overline{\boldsymbol{\sigma}}=2 \mu\left(\overline{\boldsymbol{d}}-\frac{1}{3} \bar{d}_{v} \boldsymbol{I}\right)+\bar{p} \boldsymbol{I}
$$

where the nodal deformation rate tensor $\overline{\boldsymbol{d}}$ is computed for the node $I$ as

$$
\overline{\boldsymbol{d}}^{I}=\overline{\boldsymbol{B}}^{I} \overline{\boldsymbol{v}}^{n n^{I}}
$$

where vector $\overline{\boldsymbol{v}}^{\boldsymbol{n} \boldsymbol{n}^{I}}$ contains the velocity components of node $I$ and those of its neighbor nodes (in $2 \mathrm{D}$, its size is $2 n n^{I}$, where $n n^{I}$ is the number of neighbors of node $I$ plus one). The nodal 
deformation matrix $\overline{\boldsymbol{B}}$ is computed as

$$
\overline{\boldsymbol{B}}^{I}=\left[\begin{array}{cccccccc}
N_{1, x}^{I} & 0 & \ldots & N_{I, x}^{I} & 0 & \ldots & N_{n n^{I}, x}^{I} & 0 \\
0 & N_{1, y}^{I} & \ldots & 0 & N_{I, y}^{I} & \ldots & 0 & N_{n n^{I}, y}^{I} \\
N_{1, y}^{I} & N_{1, x}^{I} & \ldots & N_{I, y}^{I} & N_{I, x}^{I} & \ldots & N_{n n^{I}, y}^{I} & N_{n n^{I}, x}^{I}
\end{array}\right]
$$

Following what proposed in [11, the derivatives of the shape functions are computed as:

$$
N_{J, j}^{I}=\frac{1}{\Omega^{I}} \sum_{\alpha=1}^{n e^{I}} N_{J, j}^{\alpha} \frac{\Omega^{\alpha}}{3}
$$

Finally, the nodal volumetric deformation rate $\bar{d}_{v}$ is computed for node $I$ as

$$
\bar{d}_{v}^{I}=\operatorname{trace}\left(\overline{\boldsymbol{d}}^{I}\right)
$$

Remark 3. Depending on the definition of the derivative of shape functions, different nodal integration schemes can be obtained (see e.g. [39]). The definition in Eq.(31) is usually referred to as nodal averaging integration. It is important to note that in the Smoothed Finite Element Method, for high order elements, the volume (or area in 2D) integration is replaced by node based integration over the patch boundaries. However, for three-node triangular elements, since the gradient of displacement is constant in each element, the integration over the patch boundaries can be simplified as node averaging (see e.g. [1, 9]). Actually, the node averaging employed in this work can be seen as a particular case of strain smoothing concept.

Remark 4. Eq.28 shows that, in a nodal approach, the two terms contributing to the computation of the stresses (pressure and strain rate) are defined at the node level. Consequently, the stress can be computed directly at the nodes without any interpolation. On the contrary, in the standard elemental FEM the two terms of Eq. (28) are defined at different positions: the pressure at nodes and the strain rate at Gauss points. To compute the stress at nodes (or at the Gauss point) one interpolation is necessary. Avoiding the interpolation step, the accuracy of the computed stress is higher and consequently, a faster convergence is expected.

\section{Stabilization}

As described in the previous sections, linear shape functions are used for the approximation of both pressure and velocity fields. It is well known that this combination does not fulfill the so-called 
inf - sup condition [23], and, although nodal integration can be beneficial for alleviating volumetric locking [1, this is not enough to guarantee the solution stability of fully-incompressible problems.

In this work, we overcome this issue by stabilizing the formulation with the so-called Finite Increment Calculus (FIC) 24] method, an assessed stabilization procedure in the framework of the PFEM formulation for incompressible fluid dynamics. The stabilizing technique was originally derived and validated in [24], and later used in several other PFEM formulations, e.g. in [30] for coupled-thermal problems, in [31] for fluid-structure interaction applications, and in [32] for nonNewtonian fluid analysis. The FIC stabilization method has shown to possess very good mass preservation properties in all its applications. Another good feature of the method is its reduced intrusivity. Indeed, the FIC stabilization terms are only included in the continuity equation, while the momentum equation remains unchanged. For these reasons, the FIC method has been employed also in this PFEM formulation with nodal integration. As in the original work 24, the stabilization terms are here assembled elementally.

To avoid repetitions of previous publications, the FIC stabilization terms are not derived in this work but are directly added to the continuity equation. The readers are invited to see the mentioned works for further details on the FIC method.

The continuity equation (Eq.227) ) is modified by adding the stabilization term $\boldsymbol{F}^{\boldsymbol{\tau}}$ as follows

$$
\boldsymbol{R}_{c}=\boldsymbol{F}^{p}-\boldsymbol{F}^{v}+\boldsymbol{F}^{\tau}=\mathbf{0}
$$

being

$$
\boldsymbol{F}^{\boldsymbol{\tau}}=\tau\left(\boldsymbol{L} \overline{\boldsymbol{p}}+\boldsymbol{M}_{\Gamma} \overline{\boldsymbol{p}}+\boldsymbol{f}_{p}\right)
$$

where matrices $\boldsymbol{L}$ and $\boldsymbol{M}_{\Gamma}$, vector $\boldsymbol{f}_{p}$, and the stabilization parameter $\tau$ are defined below following $[24$.

The stabilization parameter $\tau$ is computed element by element and at each non-linear iteration as

$$
\tau=\left(\frac{8 \mu}{h^{2}}+\frac{2 \rho}{\delta}\right)^{-1}
$$

where $h$ and $\delta$ are characteristic distances in space and time that depend on the element size and the time step increment, respectively [24].

Remark 5. For simplicity and due to their minor effect, the stabilization terms of pressure acceleration used in 24] are not considered in this work. 
Matrix $\boldsymbol{L}$ of Eq. (34) comes from the discretization of a Laplacian and is computed for each element $e$ as

$$
\boldsymbol{L}^{e}=\int_{\Omega^{e}}\left[\nabla \mathbf{N}^{e}\right]^{T} \nabla \mathbf{N}^{e} d \Omega
$$

with

$$
\nabla \mathbf{N}^{e}=\left[\begin{array}{ccc}
N_{1, x}^{e} & N_{2, x}^{e} & N_{3, x}^{e} \\
N_{1, y}^{e} & N_{2, y}^{e} & N_{3, y}^{e}
\end{array}\right]
$$

Furthermore, the components of $\boldsymbol{M}_{\Gamma}$ in 34 are defined as

$$
\left[M_{\Gamma}^{I J}\right]^{e}=\int_{\Gamma_{t}^{e}} \frac{2}{h_{n}} N^{I} N^{J} d \Gamma
$$

where $h_{n}$ is the height of the boundary triangle (tetrahedron in 3D) with respect to its free-surface edge (surface in 3D).

Finally,

$$
\left[f_{p}^{I}\right]^{e}=\int_{\Gamma_{t}^{e}} N^{I}\left[\rho \dot{v}_{n}-\frac{2}{h_{n}}\left(2 \mu d_{n}-\hat{t}_{n}\right)\right] d \Gamma-\int_{\Omega^{e}}\left[\nabla \mathbf{N}^{e}\right]^{T} \boldsymbol{b} d \Omega
$$

where $\dot{v}_{n}, d_{n}$ and $\hat{t}_{n}$ are the outer normal projections of the nodal acceleration, deformation rate and the imposed traction, respectively.

Remark 6. Each entry of $\boldsymbol{F}^{\boldsymbol{\tau}}$ in 34 is computed at each time step and at each non-linear iteration. The stabilization terms, although computed elementally, do not requireto to be re-mapped in case of remeshing, as each of them is computed directly from the updated results and over the new elemental configuration.

\section{Linearized form}

The linearized form of the momentum equations reads

$$
(\boldsymbol{M}+\boldsymbol{K}) \boldsymbol{\Delta} \overline{\boldsymbol{v}}=-\boldsymbol{R}_{m}
$$

where $\boldsymbol{\Delta} \overline{\boldsymbol{v}}$ are the increments of the nodal velocities, $\boldsymbol{R}_{m}$ is the fully discretized form of linear momentum balance (Eq. (23p), each diagonal component of the mass matrix $\boldsymbol{M}$ is computed as

$$
M_{i i}^{I}=\frac{\rho}{2 \Delta t} \Omega^{I}
$$


and the nodal stiffness matrix $\boldsymbol{K}$ is computed for each node as [1]

$$
\boldsymbol{K}^{I}=\left[\overline{\boldsymbol{B}}^{I}\right]^{T} \boldsymbol{C} \overline{\boldsymbol{B}}^{I} \Omega^{I}
$$

where matrix $\overline{\boldsymbol{B}}^{I}$ has been defined in Eq. 30 , and the constitutive matrix $\boldsymbol{C}$, in $2 \mathrm{D}$, is

$$
\boldsymbol{C}=\left[\begin{array}{ccc}
\kappa \Delta t+\frac{4 \mu}{3} & \kappa \Delta t-\frac{2 \mu}{3} & 0 \\
\kappa \Delta t-\frac{2 \mu}{3} & \kappa \Delta t+\frac{4 \mu}{3} & 0 \\
0 & 0 & \mu
\end{array}\right]
$$

Note that, due to the problem non-linearity, matrices $\boldsymbol{M}$ and $\boldsymbol{K}$ are assembled at the beginning of each of non-linear iteration considering the updated configuration.

Remark 7. For weakly compressible materials, the use of the actual bulk modulus in matrix $\boldsymbol{C}$ may worsen the conditioning of the algebraic linear system 40, which is crucial when an iterative linear solver is used. This issue was solved in [20] by scaling the fluid bulk modulus of the matrix $C$ with a reduction factor ad hoc designed to reduce the condition number of the solving matrix. As detailed in [20, the reduction of the bulk modulus in $\boldsymbol{C}$ has the unique effect of improving the conditioning of matrix $\boldsymbol{K}$ and it does not affect the accuracy of the scheme. In particular, the mass conservation properties of the scheme are still preserved (note that the bulk modulus in the continuity equation is not modified).

Remark 8. In a nodal formulation, the matrix bandwidth is larger than in an elemental approach. Consider for example the patch of Figure 1 In a nodal approach, due to the structure of the deformation matrix of node $I\left(\boldsymbol{B}^{I}\right.$, Eq. 30$)$, the non-diagonal terms of the global stiffness $\boldsymbol{K}$ referring to nodes $M$ and $P$ are in general different from zero, in contrast to an elemental approach. Being larger the matrix bandwidth of $\boldsymbol{K}$, the computational time required for the linear system solution also increases.

On the other hand, the stabilized continuity equation (Eq. (33)) is computed as

$$
\left(M_{\kappa}+L+M_{\Gamma}\right) \Delta \bar{p}=-R_{c}
$$

where $\boldsymbol{L}, \boldsymbol{M}_{\Gamma}$ and $\boldsymbol{R}_{\boldsymbol{c}}$ have been defined previously, $\boldsymbol{\Delta} \overline{\boldsymbol{p}}$ are the increments of the nodal pressures, and each nodal component of matrix $M_{\kappa}$ is computed as

$$
M_{\kappa}^{I}=\frac{1}{\kappa \Delta t} \Omega^{I}
$$

Note that in $M_{\kappa}^{I}$ the actual bulk modulus is used. 


\section{Nodal PFEM solution scheme}

The problem is solved at each time step with a two-step algorithm. Specifically, the linear momentum equations (Eq. (40) ) and the stabilized continuity equation (Eq. (44)) are solved in the updated configuration to find the increments of nodal velocities and nodal pressures, respectively. The linear momentum and continuity equations are solved iteratively until the fulfillment of the respective convergence criteria of the non-linear iterative loop. Note that, before the solution of the momentum equation, the nodal derivative of the shape functions (Eq.(31) ) and its nodal area (or the nodal volume in $3 \mathrm{D}$ ) must be computed for each node of the mesh in the current configuration.

After the solution of the time step, if the mesh is too distorted, a new discretization is built in the standard PFEM fashion [15, 16, 17. Initially, the elements of the previous mesh are erased, and all the information is stored at the node level. Based on the cloud of nodes, a new mesh is generated performing a Delaunay triangulation [22]. After that, the physical contours of the computational domain are identified using the Alpha Shape method [21. In particular, all those elements that do not fulfill the following criterion are erased from the mesh (excessively distorted or large).

$$
R<\alpha h
$$

where $R$ is the circumradius of the element, $\alpha$ is a positive parameter (typically $\alpha \simeq 1.25$ ) [18, 16, 25], and $h$ is the mean element size in the mesh.

Remark 9. A crucial advantage of the nodal integration in the PFEM framework is the possibility of a nodal definition of history variables. Typically, these variables are defined at the integration points, which, in an elemental approach, differ from the nodes. Consequently, in the classical elemental PFEM, every time the mesh is regenerated, re-mapping operations from the Gauss points of the old mesh to the ones of the new mesh are necessary in order to recover the historical information. This is not the case of nodal integration where all the quantities (and also history variables) can be defined at mesh nodes avoiding expensive interpolations and also the typical error associated with these operations [10, 9].

For a generic time step $\left[{ }^{n} t ;{ }^{n+1} t\right]$ of duration $\Delta t$, the solution scheme is summarized in the pseudo-code given in Algorithm 1 .

\footnotetext{
${ }^{2}$ Tolerance has been set equal to $10^{-4}$ in all the numerical tests in this work.
} 


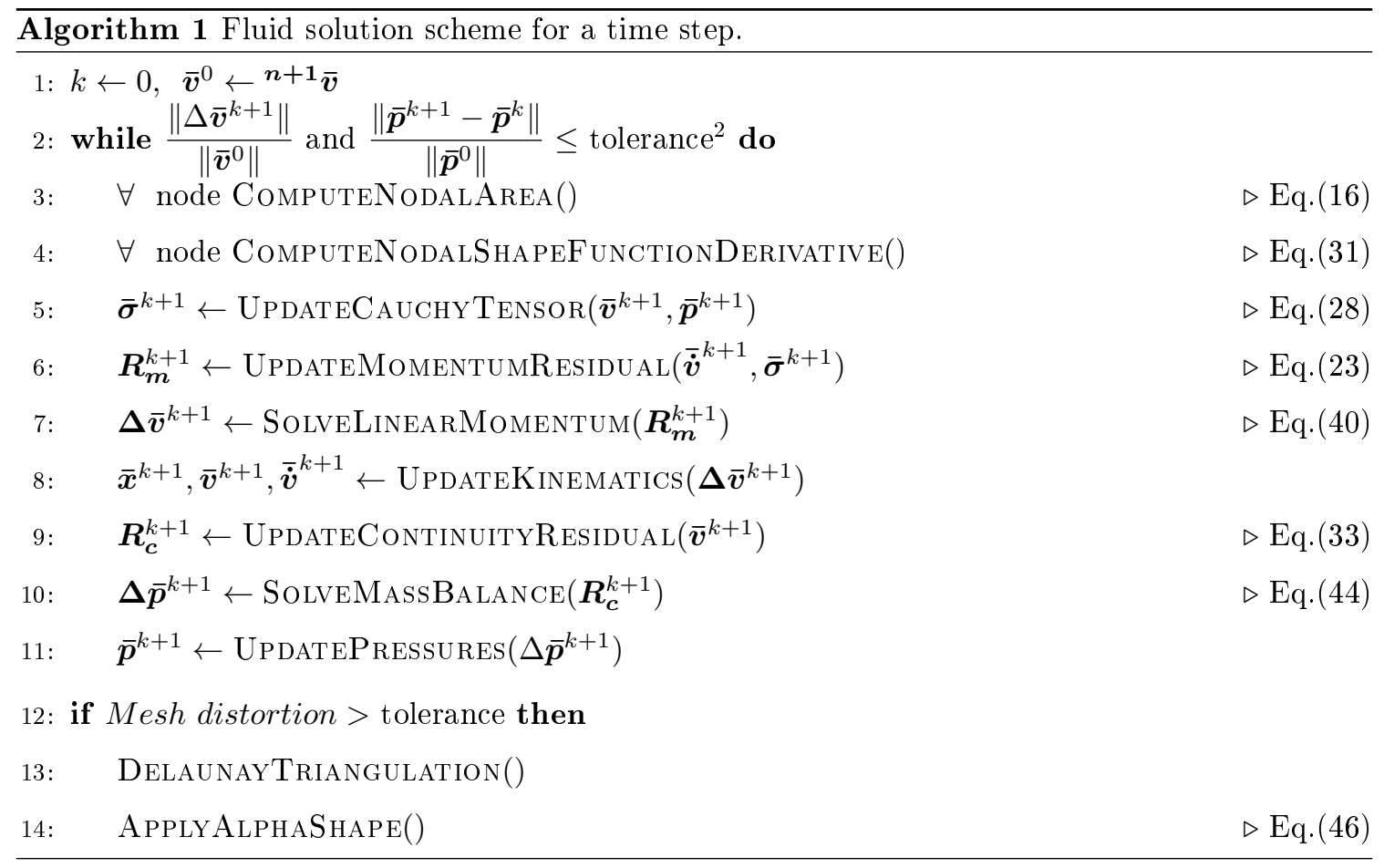

\section{Numerical examples}

In this section, several numerical tests are presented to validate the proposed nodal PFEM approach and to analyze some crucial numerical issues of the method. The first two problems have a closed analytical solution and are used to analyze the convergence behavior of the numerical formulation. The third and fourth tests are benchmark problems for free-surface fluid dynamics solvers. First, a water sloshing problem is used to analyze the convergence of the non-linear iterations (in the following, referred to as non-linear convergence) and the mass conservation properties of the nodal PFEM versus the classical elemental PFEM. For this problem, also the 3D case is analyzed. Then, a dam break test is solved for both a Newtonian and a non-Newtonian model. The accuracy of the solution is assessed by comparison to laboratory tests and to other numerical results of the literature.

\subsection{Test with analytical solution}

In this first test, we consider the two-dimensional Stokes problem with the analytical solution presented in [13]. The problem consists of determining velocity, pressure and stress fields for a 
Newtonian fluid ( $\mu=1, \rho=1, \kappa=10^{9}$ adimensional variables) occupying a square domain $\Omega=[0,1] \times[0,1]$ and submitted to the following body force per unit of volume $\boldsymbol{b}$ :

$$
\begin{aligned}
b_{x}= & (12-24 y) x^{4}+(-24+48 y) x^{3}+\left(12-48 y+72 y^{2}-48 y^{3}\right) x^{2}+ \\
& +\left(-2+24 y-72 y^{2}+48 y^{3}\right) x+1-4 y+12 y^{2}-8 y^{3}, \\
b_{y}= & \left(8-48 y+48 y^{2}\right) x^{3}+\left(-12+72 y-72 y^{2}\right) x^{2}+ \\
& +\left(4-24 y+48 y^{2}-48 y^{3}+24 y^{4}\right) x-12 y^{2}+24 y^{3}-12 y^{4}
\end{aligned}
$$

Under this prescribed body force, considering no-slip conditions at each boundary of the computational domain, the problem has the following exact stationary solution for the horizontal and vertical velocities, pressure and shear stresses, respectively

$$
\begin{aligned}
& v_{x}=4 y^{3}-6 y^{2}+x^{2}(1-x)^{2} 2 y, \\
& v_{y}=4 x^{3}-6 x^{2}-y^{2}(1-y)^{2} 2 x, \\
& p=-(1-x) x, \\
& \tau_{x y}=2\left(1-6 y+6 y^{2}\right)(1-x)^{2} x^{2}-2\left(1-6 x+6 x^{2}\right)(1-y)^{2} y^{2}
\end{aligned}
$$

The convergence analysis has been performed for five different meshes with mean size $h$ of $0.2,0.1$, 0.05, 0.025 and 0.0125 , leading to 48, 204, 880, 3596 and 14586 3-noded triangular elements, and 37, 125 482, 1881, and 7465 nodes, respectively.

The following $L-2$ norm has been used as error measures

$$
\left\|a-a_{h}\right\|=\left(\int_{\Omega}\left(a-a_{h}\right)^{2} d \Omega\right)^{1 / 2}
$$

where $a$ and $a_{h}$ are the analytical and numerical solution of the considered variable. For comparison purposes, the same problem has been solved also with the PFEM with the elemental integration presented and validated in [24].

The stationary numerical solution (reached after a normalized time of around 0.1) is shown in Figure 2, In the left column, the numerical contours (horizontal and vertical velocities, pressure and shear stress) are plotted over the whole computational domain. In the graphs of the right column, the numerical solution is compared to the analytical one (Eq. 48 ) at a transversal line of the domain.

The graphs show a perfect overlapping between the numerical and analytical solutions. The convergence analysis is shown in Figure 3 where also the results obtained with the elemental formulation [24] are plotted. 
The graphs show that the elemental and the nodal formulations have the same convergence rate (almost quadratic) for the velocity and the pressure fields (Figures 3a- 3c). However, for the same mesh, the elemental formulation gives a lower error with respect to the nodal one. On the other hand, the nodal formulation shows a much better convergence rate and lower error for the shear stress versus the elemental approach (Figure 3d). In this case, the convergence rate is almost linear for the elemental integration, whereas is practically quadratic for the nodal one.

It is also interesting to analyze the results plotted in Figure 4 where the vertical velocities at $(x, y)=(0.75,0.5)$ obtained for different meshes are shown. The graph shows that the nodal formulation gives an upper bound solution, contrary to the elemental integration scheme. In other words, in a nodal approach, the coarser is the mesh, the less stiff (or viscous) is the solution. These results confirm the ones obtained in [4, 6] in the context of non-linear solid mechanics. 
(a) $v_{x}$

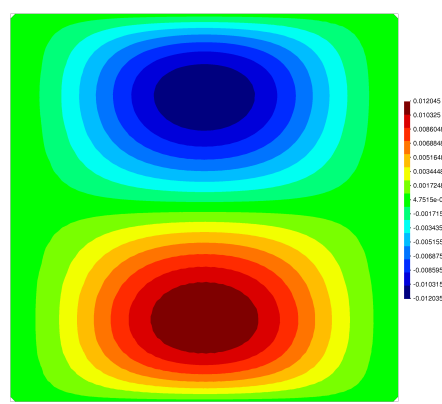

(c) $v_{y}$

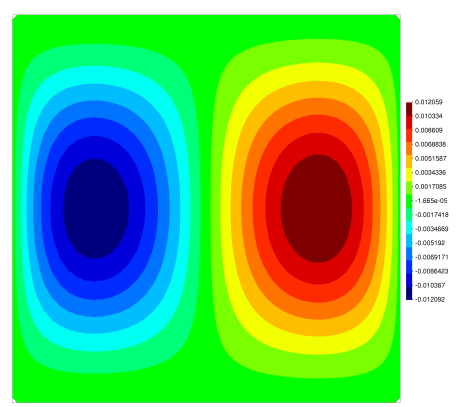

(e) $p$

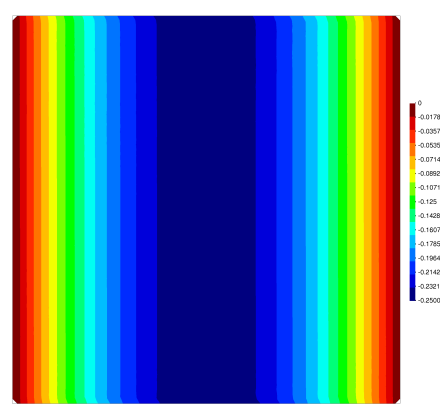

(g) $\tau_{x y}$

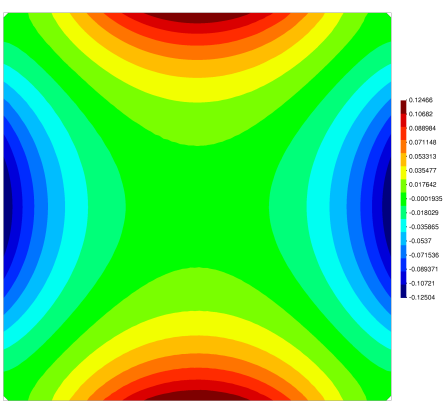

(b) $v_{x}$ at $\mathrm{x}=0.5$

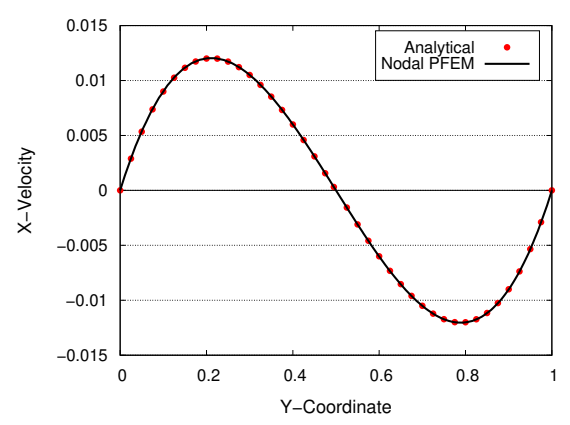

(d) $v_{y}$ at $\mathrm{y}=0.5$

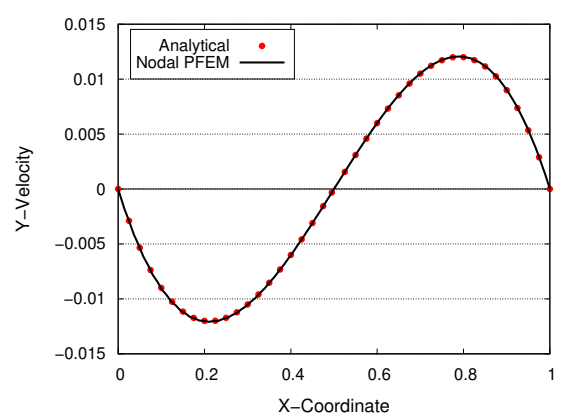

(f) $p$ at $\mathbf{y}=0.5$

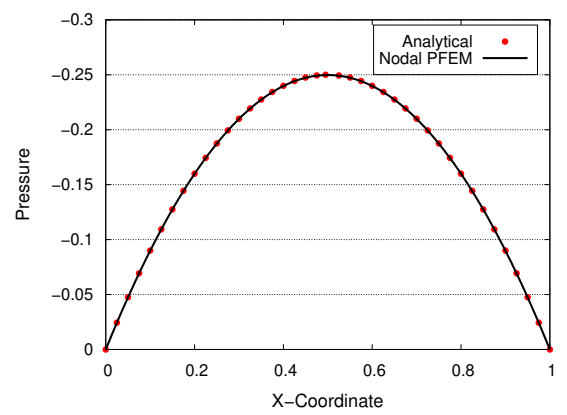

(h) $\tau_{x y}$ at $\mathbf{y}=0.5$

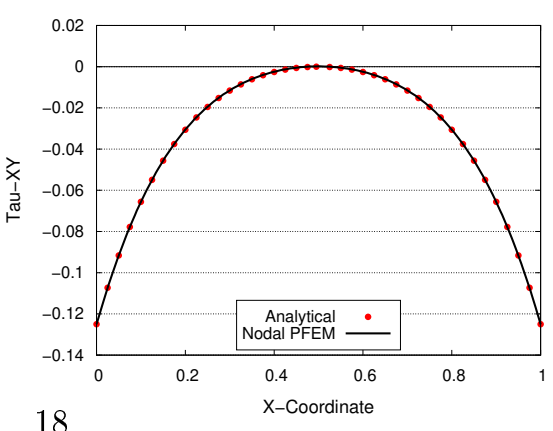

18

Figure 2: Test with analytical solution. Left column: numerical results over the whole domain. Right column: numerical and analytical results (Eq. $(48)$ ) at a transversal line of the domain. 
(a) $v_{x}$

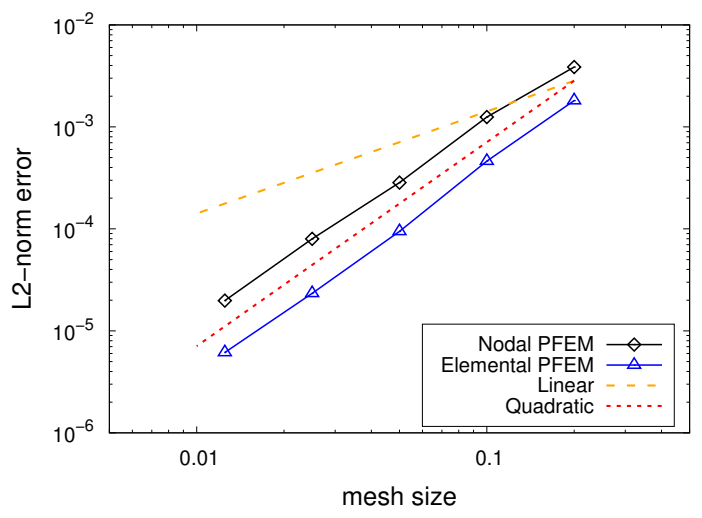

(c) $p$

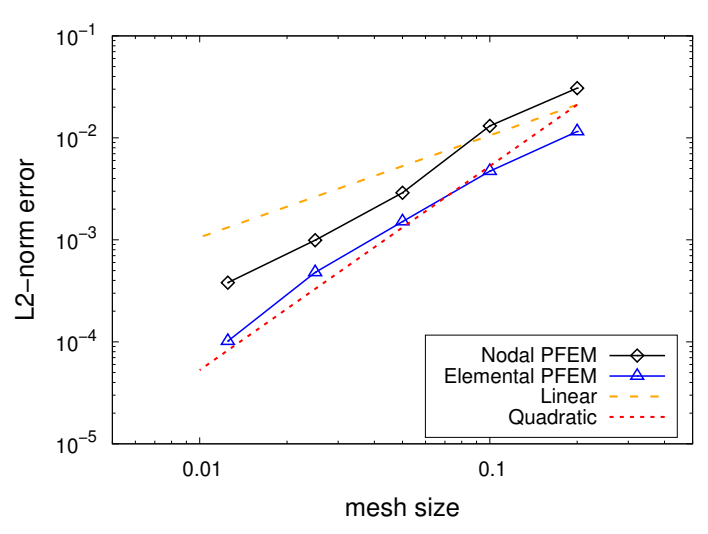

(b) $v_{y}$

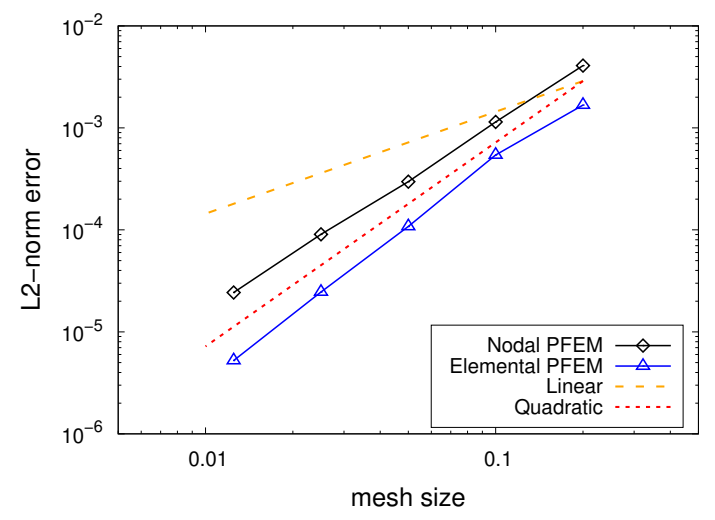

(d) $\tau_{x y}$

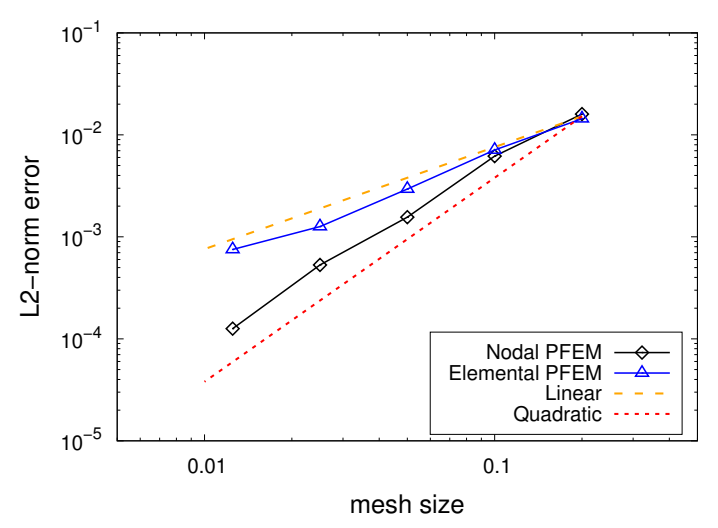

Figure 3: Test with analytical solution. Convergence graphs for nodal and elemental [24] formulations. 


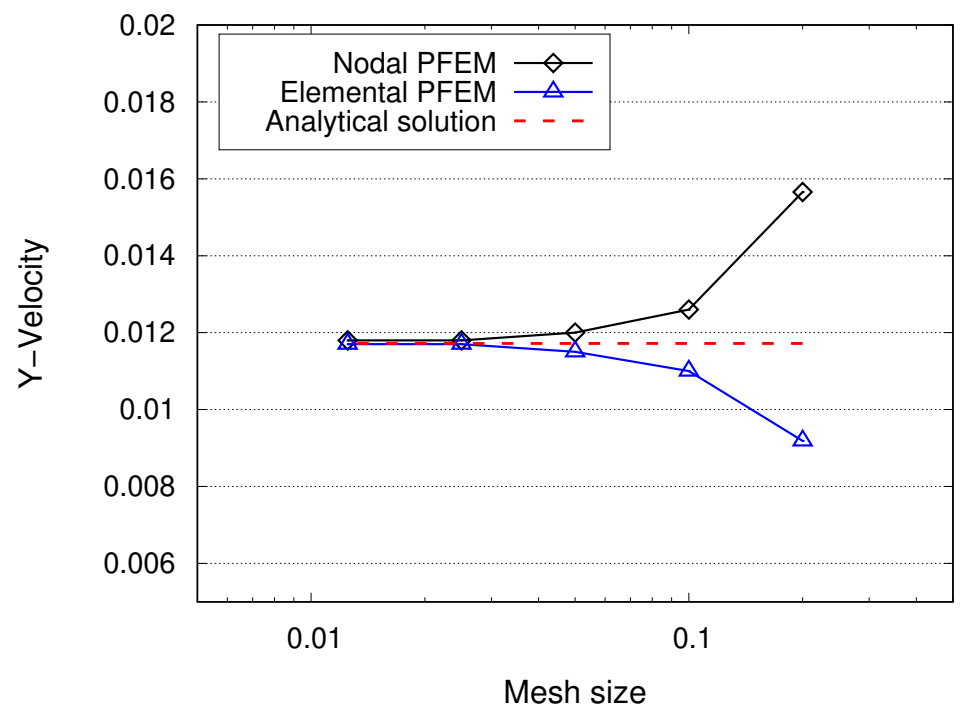

Figure 4: Test with analytical solution. Vertical velocity solution at $(x, y)=(0.75,0.5)$ for elemental and nodal formulations for different meshes. The dashed line is the analytical solution obtained with Eq. 48. 


\subsection{Circular Couette test}

In this second test, we consider the steady circular Couette flow of a Newtonian fluid [14]. The inner cylinder is rotating at a fixed angular velocity $\omega$, while the outer one is kept fixed. No-slip conditions are considered for all the solid boundaries, and the gravitational acceleration is zero. Assuming the two cylinders to be infinitely long, the hoop velocity $v_{\theta}$ and the shear stress $\tau_{r \theta}$ are expected to be

$$
\begin{aligned}
& v_{\theta}(r)=\frac{\beta^{2} \omega R}{1-\beta^{2}}\left(\frac{R}{r}-\frac{r}{R}\right) \\
& \tau_{r \theta}(r)=\frac{2 \mu \beta^{2} \omega R^{2}}{1-\beta^{2}} \frac{1}{r}
\end{aligned}
$$

where $r$ is the radial position with respect to the center of the cylinder, $R$ the radius of the outer cylinder and $\beta$ is defined such that $\beta R$ is the radius of the inner cylinder. The geometry of the problem together with an example of the 3 -noded triangular mesh used are depicted in Figure 5
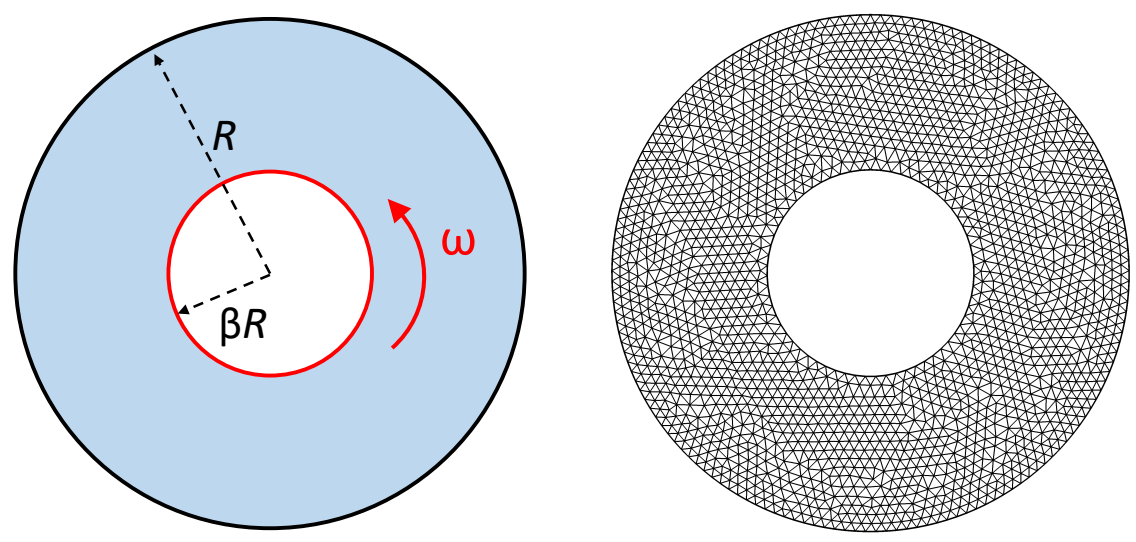

Figure 5: Circular Couette test. Initial geometry and mesh with mean size $h=0.02$.

In our analysis, a Newtonian fluid with viscosity $\mu=10^{2}$, density $\rho=10^{3}$ and bulk modulus $\kappa=10^{9}$, and the geometrical parameters $R=0.5$ and $\beta=0.4$ are considered. As for the previous case, also in this problem the stationary solution (Eq. $(50)$ is reached after a transient phase. The stationary solution (captured at a normalized time of $t=1$ ) is plotted in Figure 6 . The pictures of the left column shows the numerical solutions for $v_{\theta}$ and $\tau_{r \theta}$, while in the graphs of the right column, the numerical and analytical (Eq. 50 ) solutions at $y=0$ are plotted.

The results show a perfect match between the numerical and analytical solutions, confirming the 
accuracy of the proposed nodal integration scheme. As for the previous test, also the convergence is analyzed (the error is computed according to Eq. (49)). Five different meshes have been considered with the following mean mesh sizes: $0.005,0.01,0.02,0.04$ and 0.08 . The number of nodes of each one of these meshes is $30721,7762,1957,494$, and 130, respectively. The convergence graphs for $v_{\theta}$ and $\tau_{r \theta}$ are plotted in Figure 7

As for the previous analytical test, the velocity field shows a quadratic convergence. The shear stress $\tau_{r \theta}$ shows again an almost quadratic convergence rate.

It is also interesting to analyze the effect of the PFEM remeshing over the solution accuracy. For this purpose, the error in time computed with and without remeshing is compared for both the nodal and elemental integration schemes. Only the intermediate mesh (mean mesh size 0.02) is considered in this study. In Figure 8, the time evolution of the error for $v_{\theta}$ and $\tau_{r \theta}$ obtained using the nodal and the elemental PFEM, with (solid lines) and without (dashed lines) remeshing, is shown.

The results of both nodal and elemental formulations show that the PFEM remeshing, although inducing a certain perturbation of the numerical solution, does not jeopardize the quality of the numerical results. The graphs show that the error keeps almost constant despite the continuous remeshing operations done with the PFEM. This is clear evidence of the efficiency of this Lagrangian method for large deformation problems. Furthermore, the results of Figure 8 show that, without remeshing, the error grows faster with the elemental approach than with a nodal formulation. It can be concluded that the nodal approach is less affected by the mesh distortions. This, besides confirming the literature results [8], represents an important advantage of the nodal approach in the PFEM framework, as it allows to reduce the number of the required remeshing events in a PFEM analysis. On the other hand, Figure $8 \mathrm{~b}$ shows that the oscillations suffered by the shear stress error and induced by the remeshing are slightly larger in the nodal approach than in the elemental one. 
(a) $v_{\theta}$

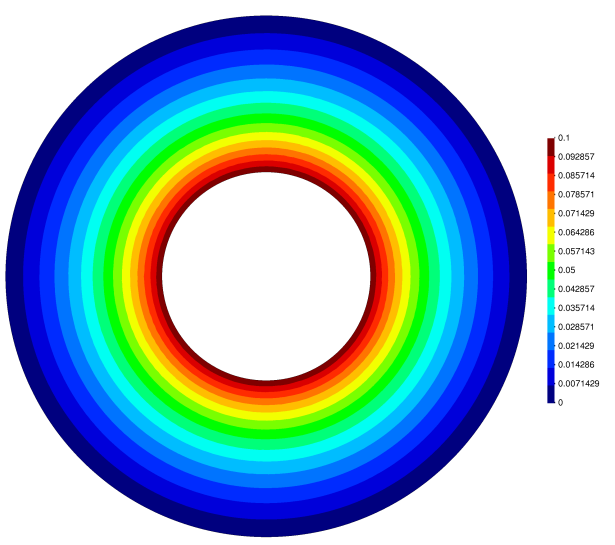

(c) $\tau_{r} \theta$

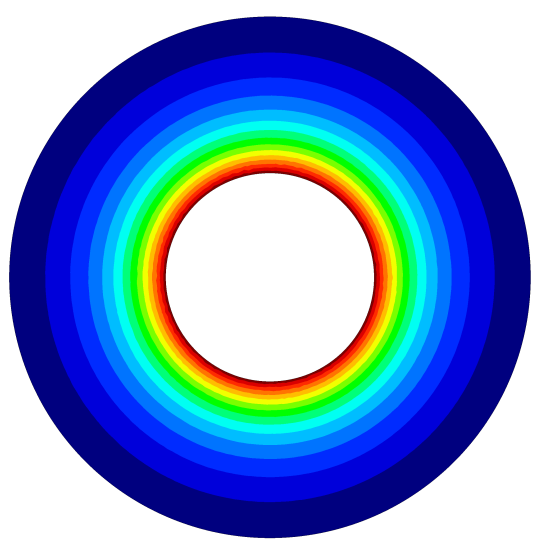

(b) $v_{\theta}$ at $\mathrm{y}=0$

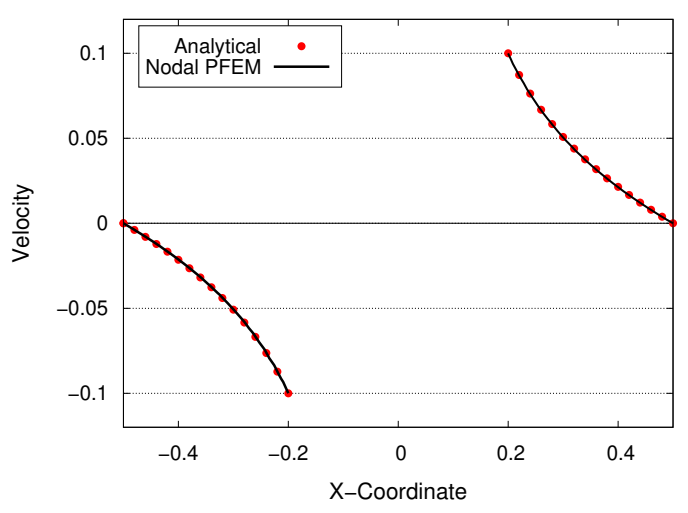

(d) $\tau_{r \theta}$ at $\mathrm{y}=0$

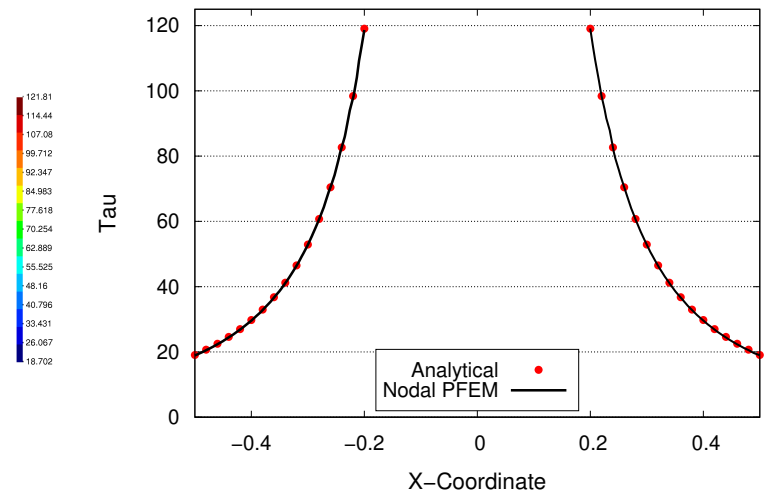

Figure 6: Circular Couette test. Left column: numerical results obtained with the proposed method. Right column: numerical and analytical (Eq. 50 ) results at $y=0$. 
(a) $v_{\theta}$

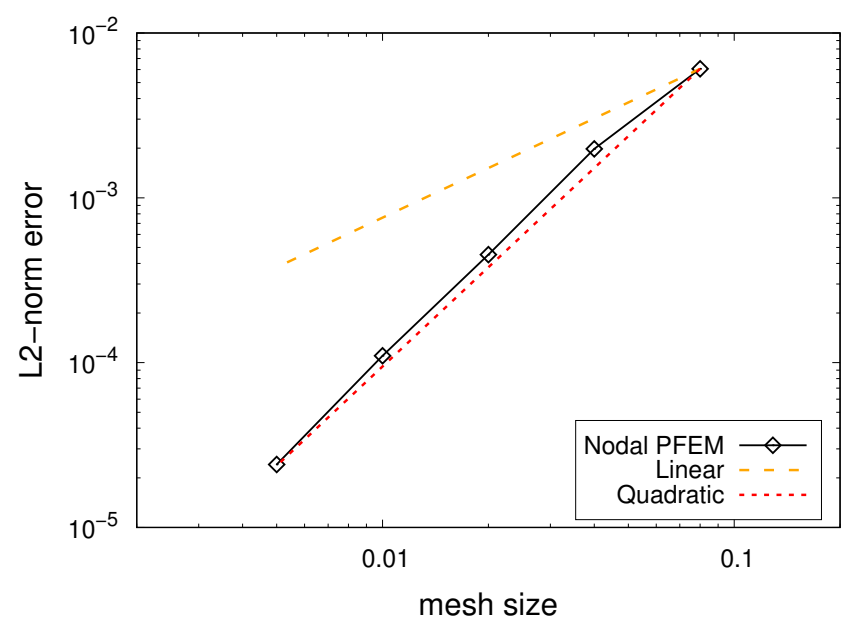

(b) $\tau_{r \theta}$

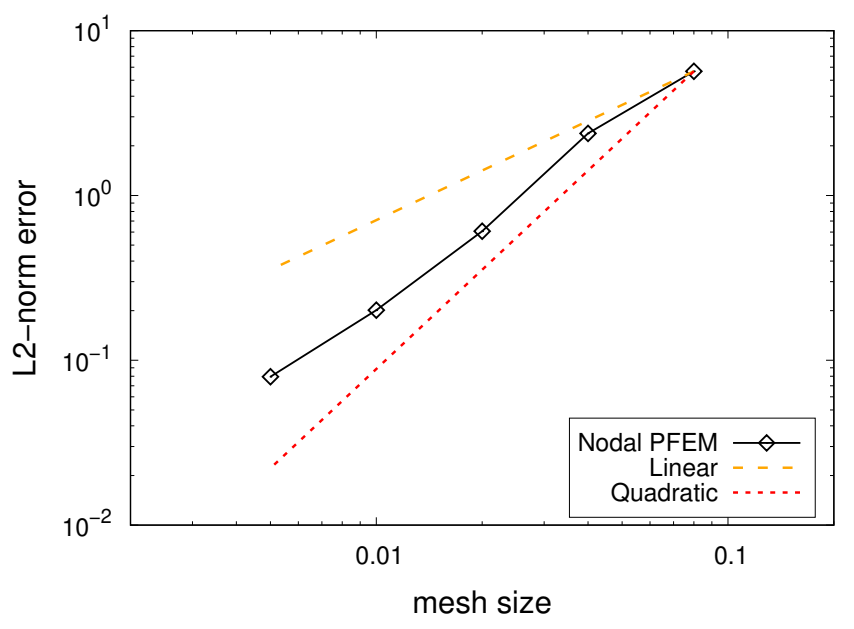

Figure 7: Circular Couette test. Convergence graphs. 
(a) $v_{\theta}$

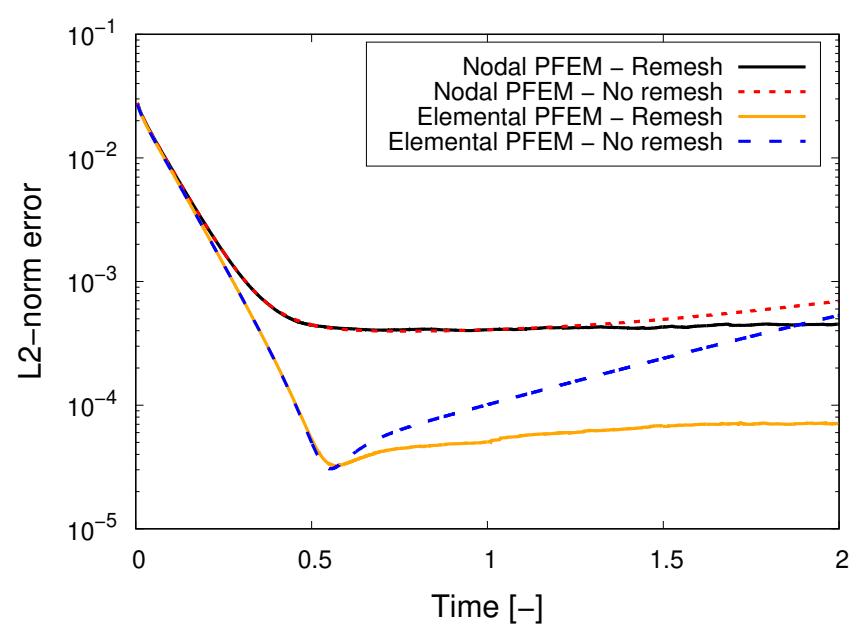

(b) $\tau_{r \theta}$

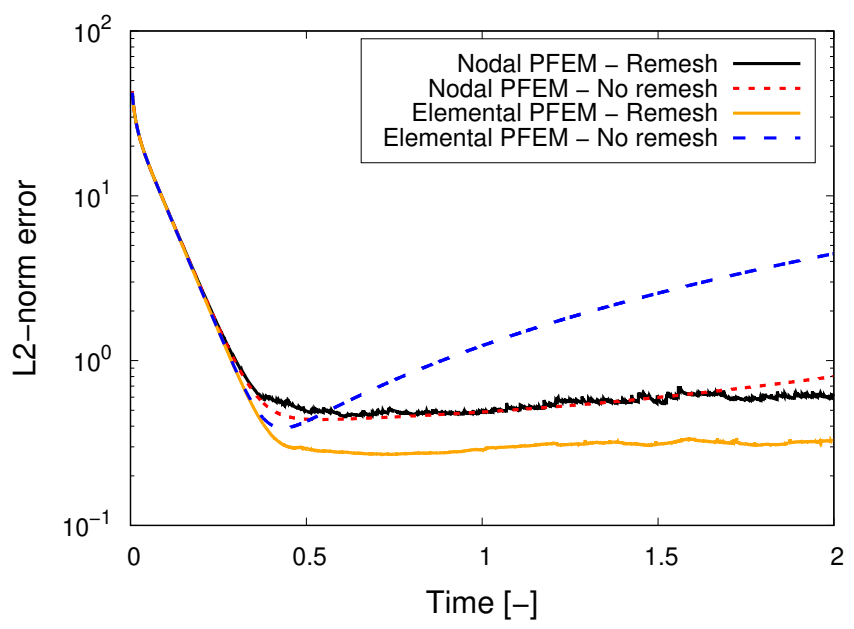

Figure 8: Circular Couette test. Time evolution of solution error for elemental [24] and nodal PFEM formulations. 


\subsection{Water sloshing}

The proposed nodal approach is tested here in a water sloshing problem; a benchmark test for free-surface fluid solvers. The initial geometry of the problem is given in Figure 9. The water

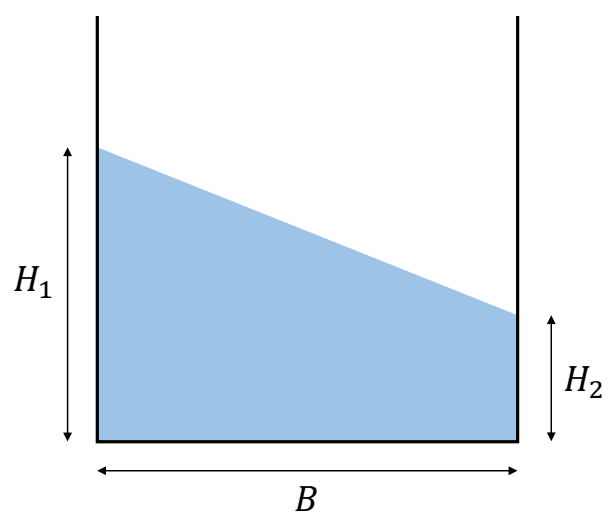

Figure 9: Water sloshing. Initial geometry $\left(B=0.5 \mathrm{~m}, H_{1}=0.35 \mathrm{~m}\right.$ and $\left.H_{1}=0.15 \mathrm{~m}\right)$.

$\left(\mu=0.001 \mathrm{~Pa} \cdot \mathrm{s}, \rho=1000 \mathrm{~kg} / \mathrm{m}^{3}, \kappa=2.1 \cdot 10^{9} \mathrm{~Pa}\right.$ ) has been discretized with 5066 3-noded triangular elements (2703 nodes), with mean mesh size of $7.5 \mathrm{~mm}$. Non-slip conditions have been considered at the rigid boundaries and a fixed time step of $0.0005 \mathrm{~s}$ has been used for all the analyses. The numerical solution is validated and compared against the elemental solution obtained with the PFEM formulation presented in [24] using the same time step and meshes of nodal integration case. The pressure field obtained at three different time steps by the nodal and elemental PFEMs are plotted in Figure 10

The pictures show a very good agreement between the two solutions. Furthermore, a smooth pressure field is obtained in both formulations, confirming the efficiency of the FIC stabilization technique.

For this problem, also the non-linear convergence has been analyzed. Figure 11 shows the nonlinear convergence of the momentum and continuity equations for the same time instants considered in Figure 10. The results of both nodal and elemental approaches are plotted. The graphs show that the nodal and the elemental methods have a similar convergence rate in the solution of the continuity equation. This was somehow expected, considering that the two formulations use the same stabilization technique. On the other hand, the nodal formulation shows a sensibly higher convergence rate in the linear momentum solution. This result can be explained considering that 
(a) Nodal at $t=0.88 \mathrm{~s}$

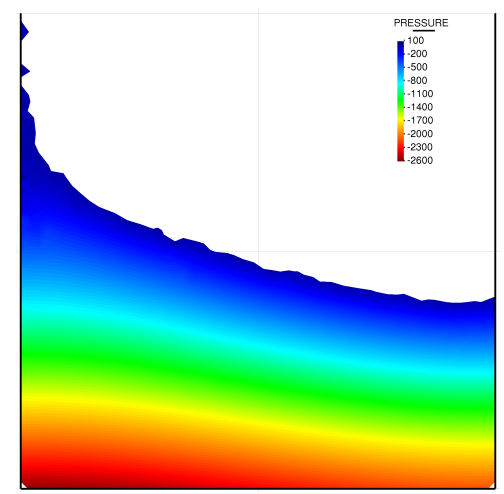

(d) Elemental at $t=0.88 \mathrm{~s}$

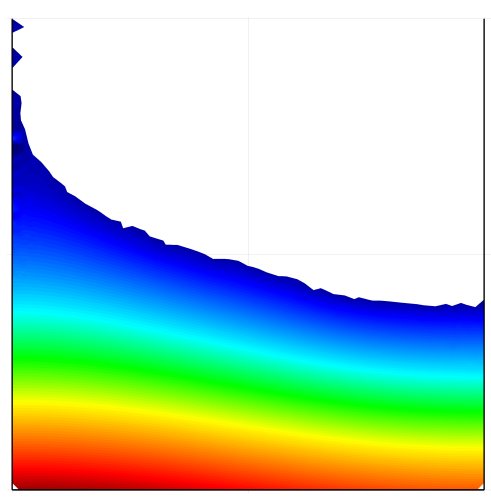

(b) Nodal at $t=2.06 \mathrm{~s}$

(c) Nodal at $t=5.00 \mathrm{~s}$

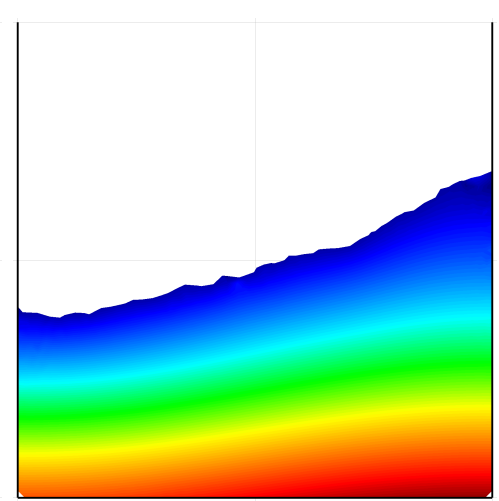

(e) Elemental at $t=2.06 \mathrm{~s}$

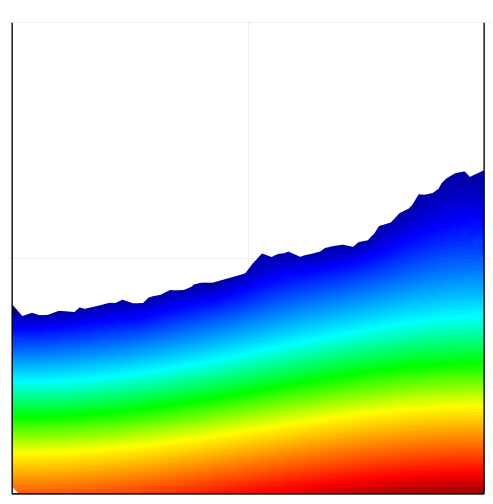

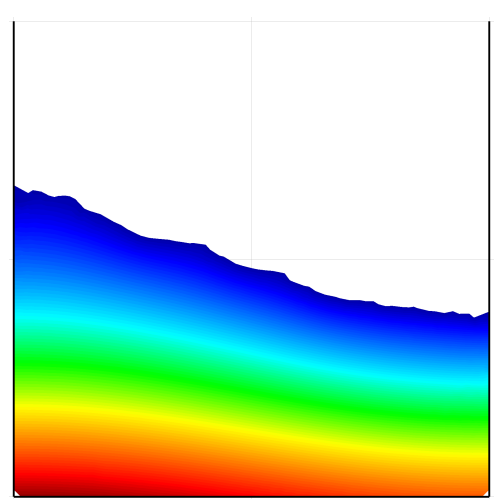

(f) Elemental at $t=5.00 \mathrm{~s}$

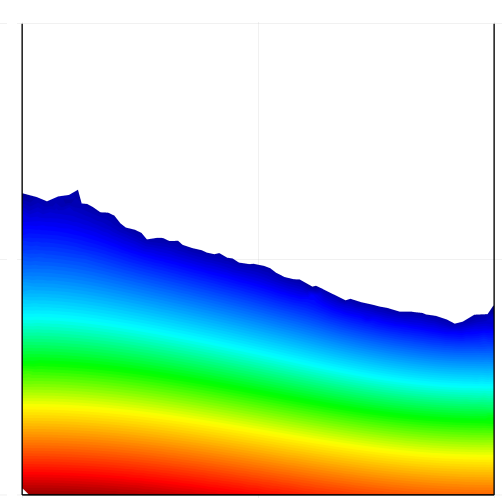

Figure 10: Water sloshing. Pressure fields at three time instants. First row: nodal PFEM. Second row: Elemental PFEM [24].

the quality of the stress is higher in the nodal approach.

For $t=5 \mathrm{~s}$, the nodal PFEM showed a slightly better non-linear convergence than the elemental PFEM. Fixing to $10^{-4}$ the tolerance for the non-linear convergence criterion of both the continuity and linear momentum equations, the nodal approach required a mean value of 4.8 iterations to converge, while the elemental one required 5.1 iterations.

To conclude, also the mass conservation properties of the proposed scheme have been analyzed. In Figure 12 the time evolution of the mass (or volume) variation induced by the numerical computation is plotted for both the nodal and elemental formulations. Note that the volume variation induced by the remeshing step is not taken into account. The graphs show a very similar behavior 
between the nodal and the elemental approaches. For $t=5 \mathrm{~s}$, both methods give around $0.2 \%$ of mass losses with respect to the initial mass, a very small value for this free-surface problem.

\subsubsection{D solution}

The same sloshing problem is analyzed here in three dimensions to show the benefit of the proposed nodal PFEM formulation also for more computationally demanding problems. To compare 2D and 3D results, a sufficiently large container with a width of $0.15 \mathrm{~m}$ has been considered. The initial mesh, plotted in Figure 13, has around 268000 4-noded tetrahedra (47800 nodes).

The 3D results at the same time instants considered in Figure 10 are plotted in Figure 14 . In the first row, the 3D view is plotted, while in the second row the pressure field is plotted at the central section only (plane $z=0.075$ ).

The results show that a smooth pressure field is obtained also with the 3D analysis. Furthermore, the deformed fluid configuration and the pressure values are very similar to those obtained with the 2D plane strain analysis (Figure 10). These results confirm the consistency of the proposed nodal approach and its applicability to computationally expensive 3D problems. 
(a) Momentum at $t=0.88 \mathrm{~s}$

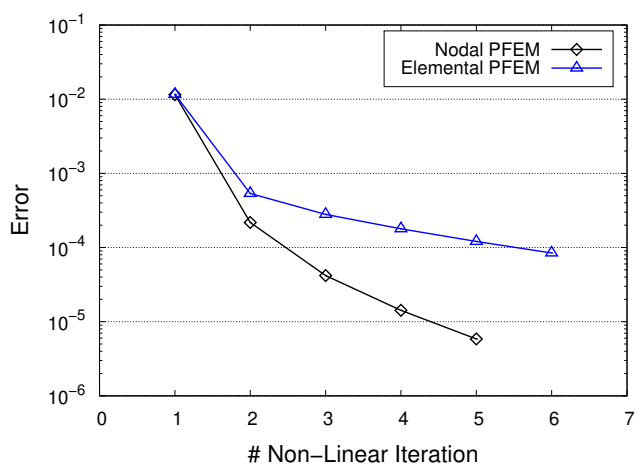

(c) Momentum at $t=2.06 \mathrm{~s}$

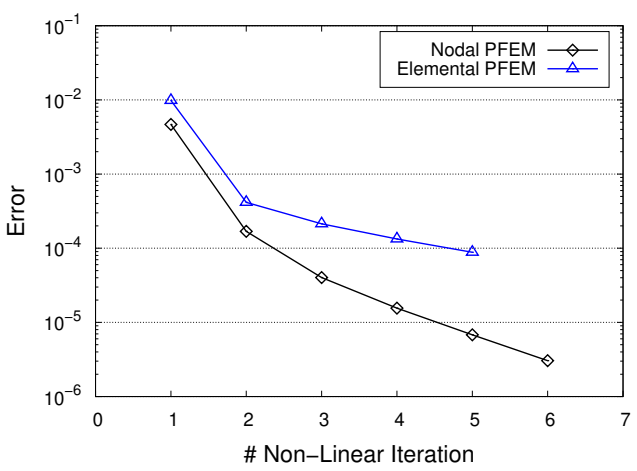

(e) Momentum at $t=5.00 \mathrm{~s}$

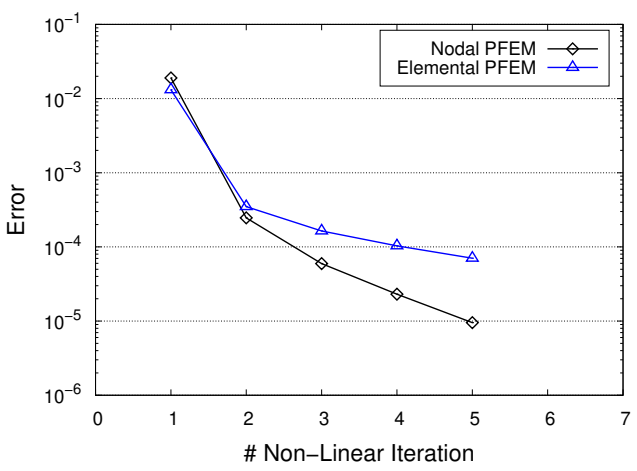

(b) Continuity at $t=0.88 \mathrm{~s}$

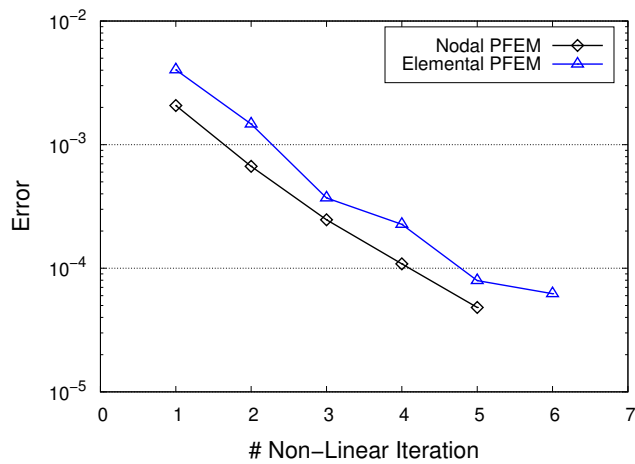

(d) Continuity at $t=2.06 \mathrm{~s}$

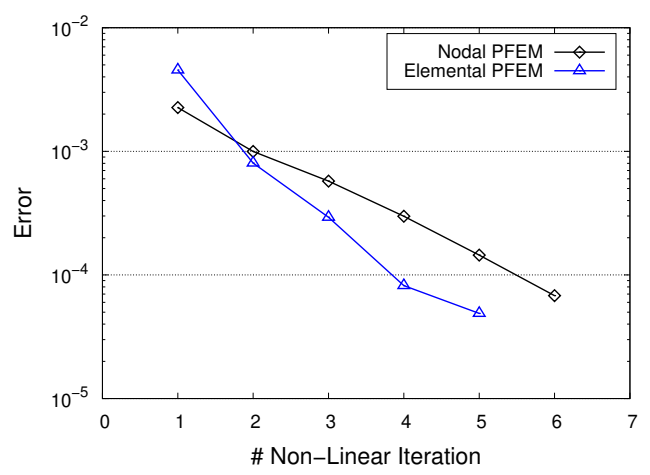

(f) Continuity at $t=5.00 \mathrm{~s}$

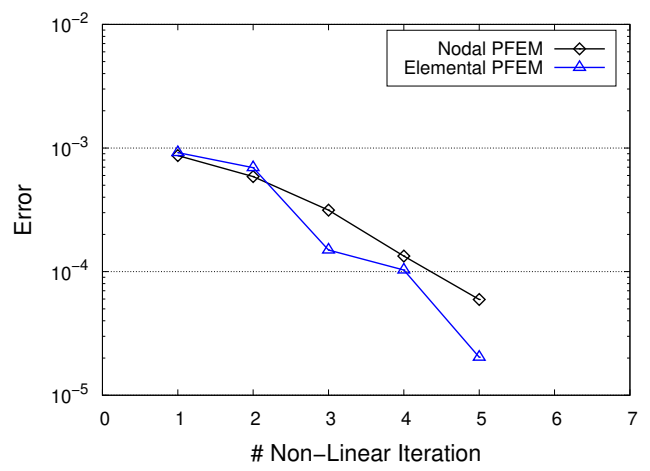

Figure 11: Water sloshing. Non-linear convergence for elemental [24] and nodal PFEM. 


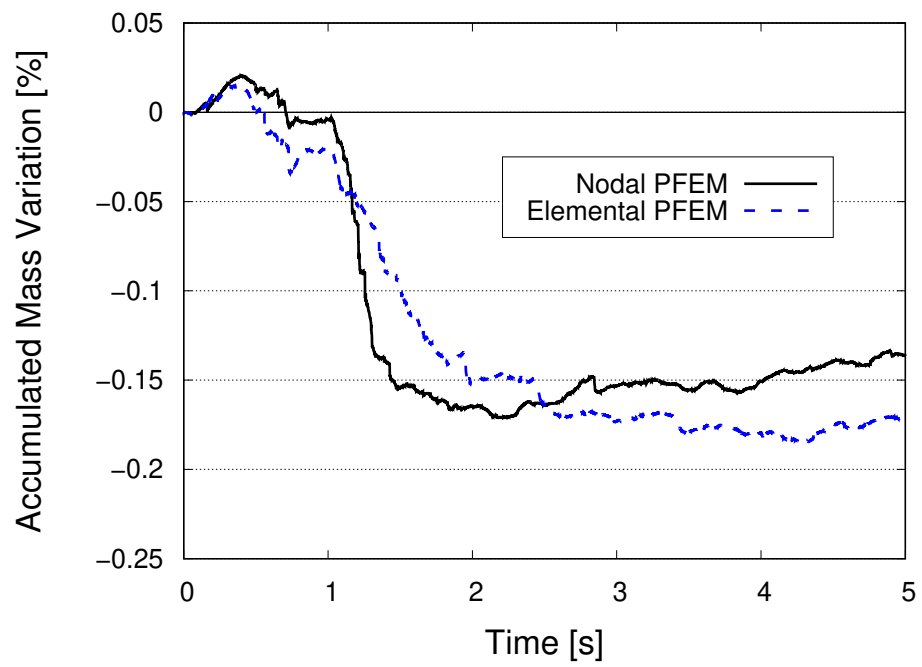

Figure 12: Water sloshing. Time evolution of the accumulated mass variation due to computation (mass variation due to remeshing is not considered). Results for the proposed nodal PFEM and the elemental PFEM [24].

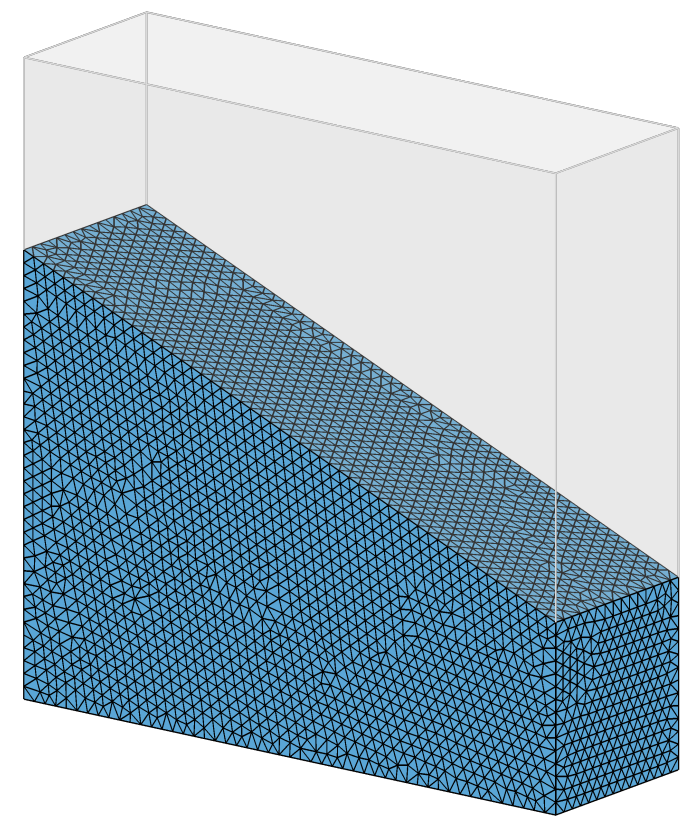

Figure 13: 3D water sloshing. Initial geometry and tetrahedral mesh. The tank width is $0.15 \mathrm{~m}$ while the other dimensions are as shown in Figure 9 for the 2D case. 
(a) $3 \mathrm{D}$ view at $t=0.88 \mathrm{~s}$

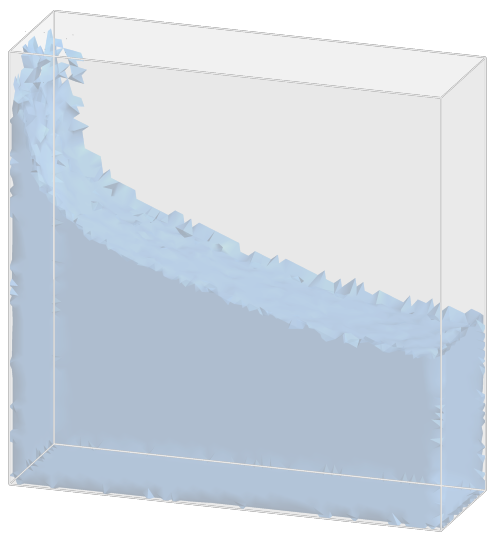

(d) Central sect. at $t=0.88 \mathrm{~s}$

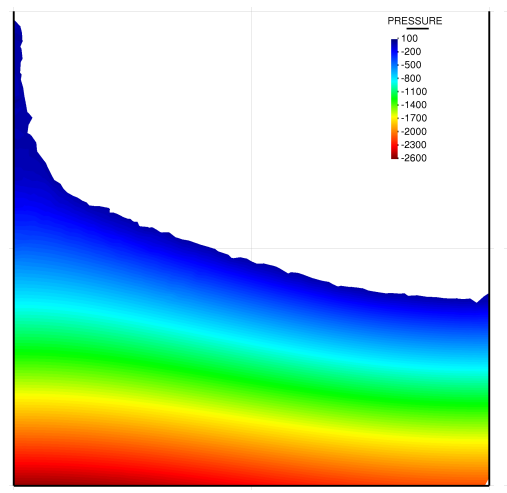

(b) $3 \mathrm{D}$ view at $t=2.06 \mathrm{~s}$

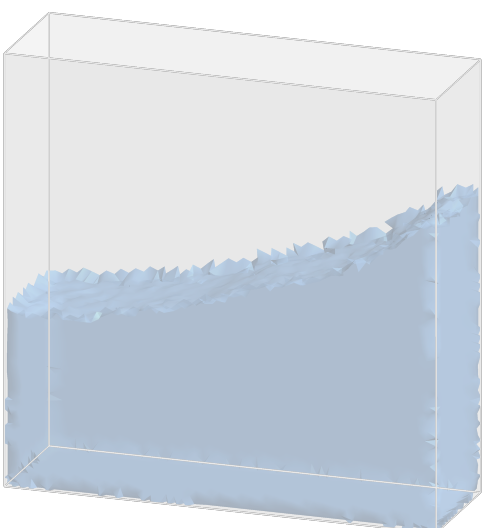

(e) Central sect. at $t=2.06 \mathrm{~s}$

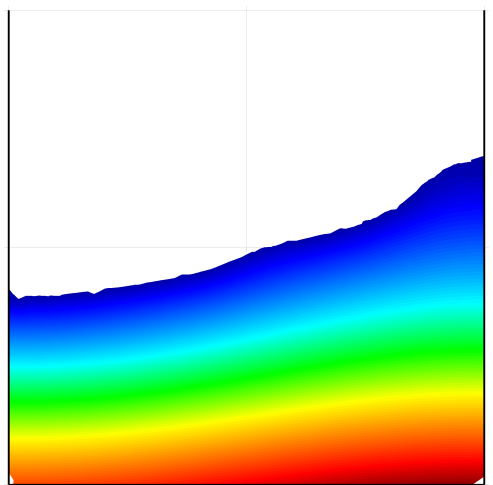

(c) $3 \mathrm{D}$ view at $t=5.00 \mathrm{~s}$

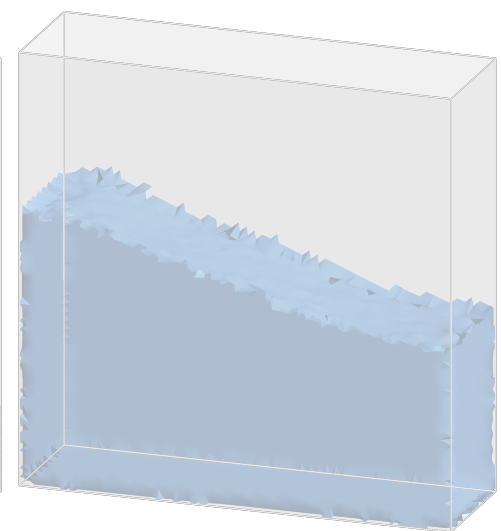

(f) Central sect. at $t=5.00 \mathrm{~s}$

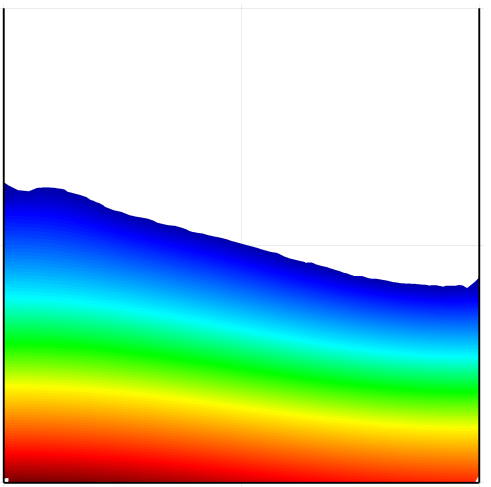

Figure 14: 3D Water sloshing. Results at three time instants. First row: 3D view. Second row: Pressure field at the central section (plane $z=0.075$ ). 


\subsection{Water dam break}

To verify the behavior of nodal PFEM in a highly unsteady free-surface fluids flow, a water dam break is here studied. The test consists of modeling the sudden collapse of a water column and its consequent flow and impact against a vertical wall. The initial geometry of the problem is depicted in Figure 15. As for the previous tests, non-slip conditions have been considered at the rigid boundaries. The column of water $\left(\mu=0.001 \mathrm{~Pa} \cdot \mathrm{s}, \rho=1000 \mathrm{~kg} / \mathrm{m}^{3}, \kappa=2.1 \cdot 10^{9} \mathrm{~Pa}\right.$ ) has

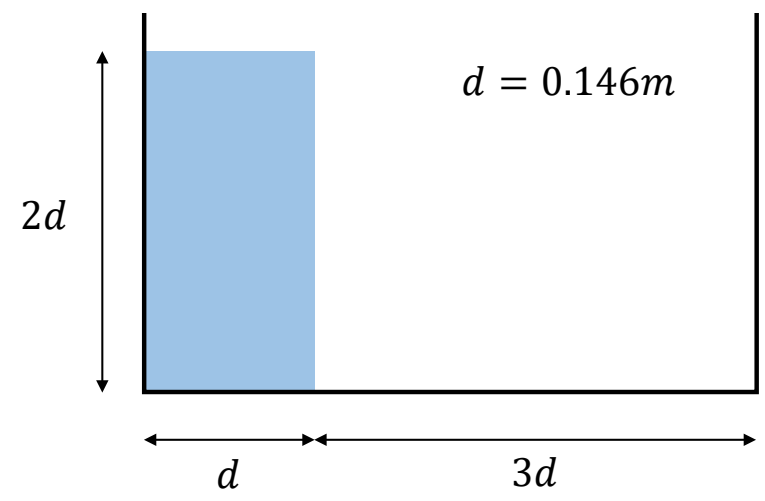

Figure 15: Water dam break. Initial geometry.

been discretized with 6112 3-noded triangular elements and 3600 nodes (mean element size of 4 $\mathrm{mm}$ ). A fixed time step of $0.00025 \mathrm{~s}$ has been used during the analysis.

In Figure 16, the numerical results obtained with the nodal PFEM are compared to those of the laboratory test presented in [26]. This qualitative comparison shows a very good agreement between the numerical and the experimental results, proving the capability of the nodal PFEM to simulate complex fluid flow problems involving impacts, splashes and breaking waves.

In Figure 17 the advancing in time of the fluid front obtained with the proposed formulation is compared to other numerical results of the literature, namely, [28] and 27, where a Moving Particle Semi-implicit method (MPS) and an explicit PFEM were used, respectively. In the graph, also the experimental observations of [26] are plotted. The following dimensionless variables are considered in the graph of Figure 17

$$
\begin{aligned}
& x^{*}=x / d \\
& t^{*}=t(2 g / d)^{0.5}
\end{aligned}
$$

where $x$ is the horizontal coordinate (at the beginning $x=0.146 \mathrm{~m}$ ) and $g=9.81 \mathrm{~m} / \mathrm{s}^{2}$ is the gravity. 
The plots show that the proposed nodal PFEM yields a solution similar to those obtained by the other computational methods. However, all the numerical simulations seem to overestimate the velocity of the fluid front obtained experimentally. As already noted in [27] and [29], this may be due to the fact that, in the numerical simulations, the retaining wall is removed instantaneously, while, in the experiments, this action takes inevitably a certain time. Nevertheless, also under this respect, the comparison with the experimental results can be considered satisfactory. 
(a) Nodal PFEM at $t=0.2 \mathrm{~s}$

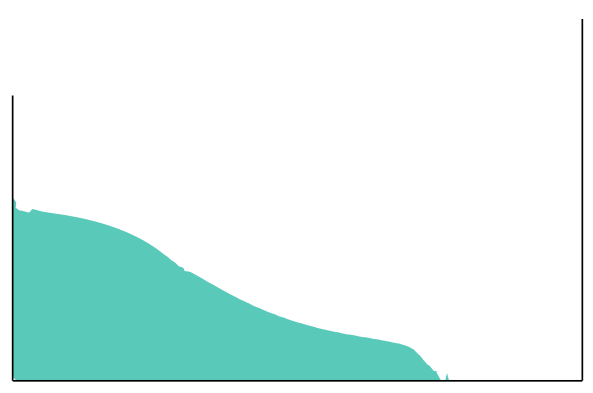

(c) Nodal PFEM at $t=0.4 \mathrm{~s}$

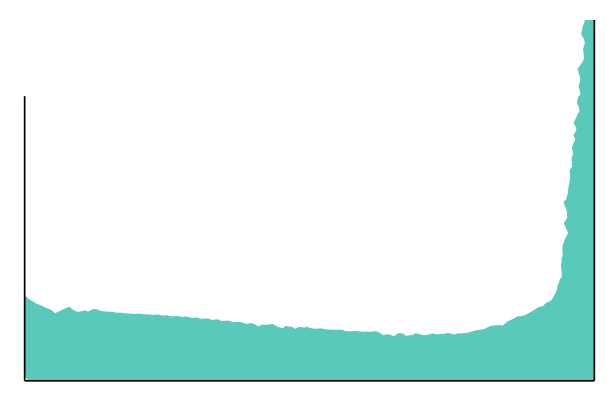

(e) Nodal PFEM at $t=0.6 \mathrm{~s}$

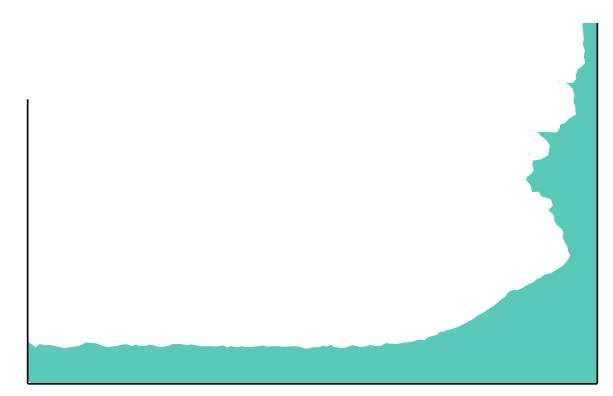

(g) Nodal PFEM at $t=0.8 \mathrm{~s}$

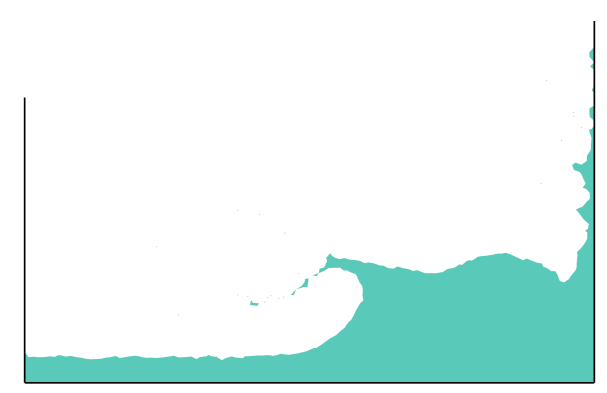

(b) Experiment [26] at $t=0.2 \mathrm{~s}$

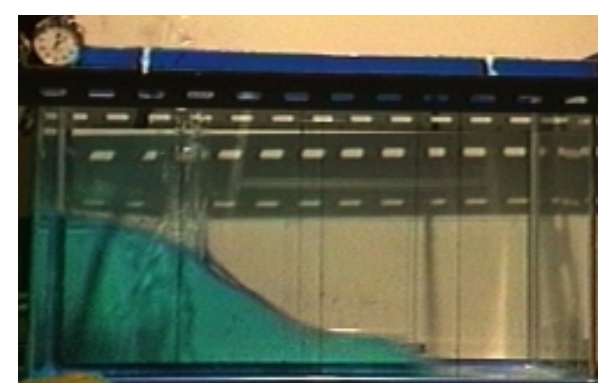

(d) Experiment [26] at $t=0.4 \mathrm{~s}$

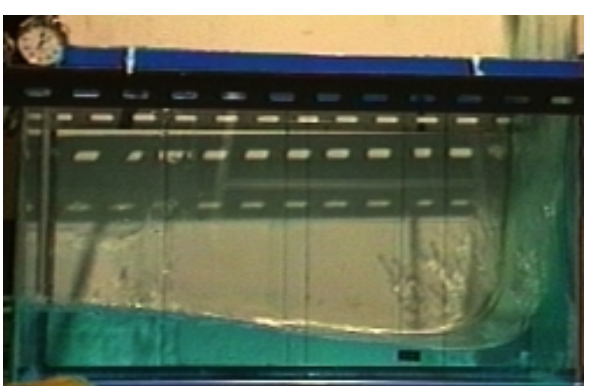

(f) Experiment [26] at $t=0.6 \mathrm{~s}$

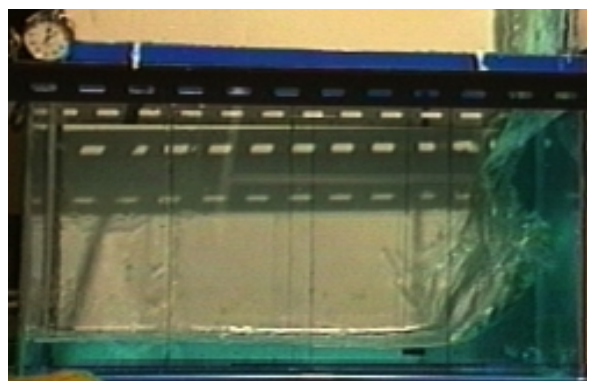

(h) Experiment [26] at $t=0.8 \mathrm{~s}$

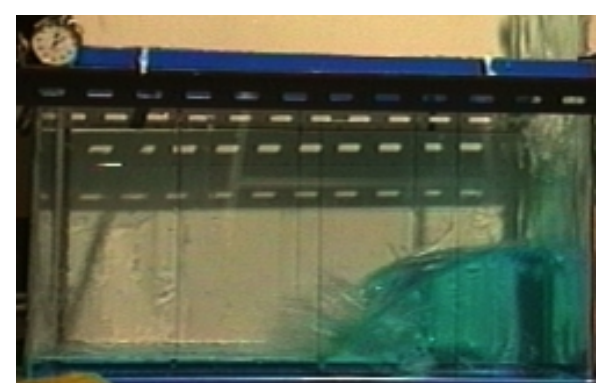

Figure 16: Water dam break. Left column: numerical results of the proposed nodal PFEM. Right column: experimental results from [26] at synchronized time instants. 


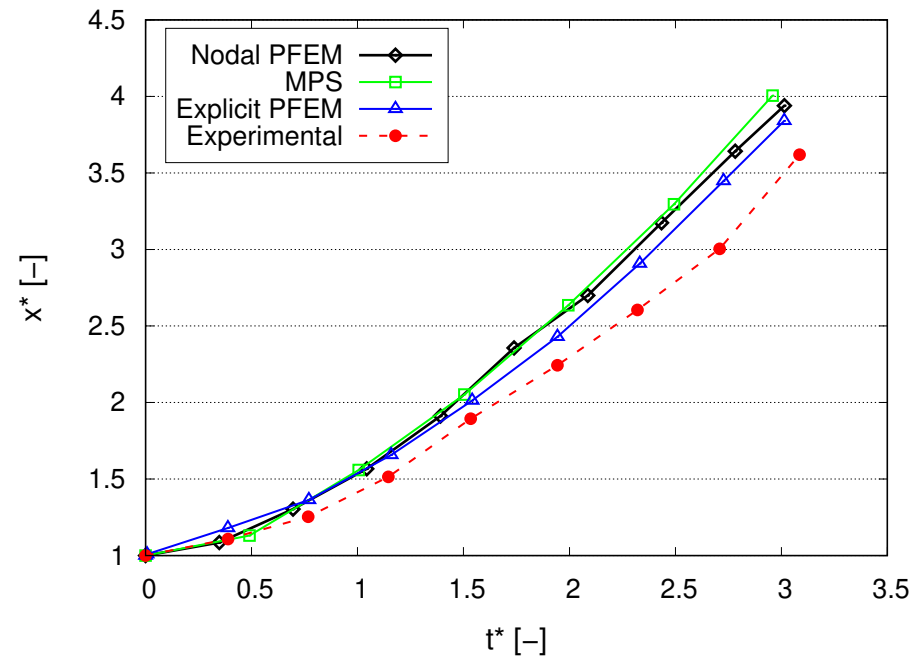

Figure 17: Water dam break. Dimensionless time evolution of the front wave position obtained with the nodal PFEM. Other results: MPS from Koshizuka et al. [28], explicit elemental PFEM from Meduri et al. [27], and experimental results from Koshizuka et al. [26]. 


\subsection{Dam Break of a non-Newtonian fluid}

The same geometry and mesh of the previous test are here used to simulate the dam break of a non-Newtonian fluid. This test is used to verify the applicability of the nodal PFEM to more complex constitutive laws in which the accuracy of the strain field is of paramount importance because it affects directly the constitutive parameters (in this case, the apparent viscosity of the non-Newtonian model).

In this example, a regularized form of the Bingham law [42, has been considered. According to this model, the nodal deviatoric stresses are computed as

$$
\overline{\boldsymbol{\sigma}^{\prime}}=2\left[\mu+\frac{\tau_{0}}{|\dot{\bar{\gamma}}|}\left(1-e^{-m|\dot{\gamma}|}\right)\right] \overline{\boldsymbol{d}}^{\prime}
$$

where $m$ is a regularization parameter, $\tau_{0}$ is the shear yield stress, and $|\dot{\bar{\gamma}}|=\left(2 \overline{\boldsymbol{d}}^{\prime}: \overline{\boldsymbol{d}}^{\prime}\right)^{\frac{1}{2}}$ is the equivalent strain rate.

The problem is analyzed for two non-Newtonian fluids which differ for the shear yield stress, namely, $\tau_{0}=300 P a$ and $\tau_{0}=400 P a$. The remaining constitutive parameters are equivalent in the two cases: $\rho=1000 \mathrm{~kg} / \mathrm{m}^{3}, \mu=1 \mathrm{~Pa} \cdot \mathrm{s}, \kappa=2.1 \cdot 10^{9} \mathrm{~Pa}$, and $\mathrm{m}=1000 \mathrm{~s}$.

In Figure 18 six snapshots of the case with $\tau_{0}=300 P a$ are given. The velocity field, with fixed contours, is plotted over the deformed fluid configuration. The pictures show the capability of the nodal PFEM to reproduce complex phenomena, such as the formation of the shear band and the arrest of the mobilized material. Note also that, due to the high value of the shear yield stress, the top outer corner of the fluid behaves as a rigid solid rotating under the motion of the underneath material. For validation purposes, the numerical results are compared to those obtained using the elemental non-Newtonian PFEM formulation presented and validated in [32. In Figure 19, the time evolution of the dimensionless wave front position obtained by both PFEM formulations is plotted for both analyzed fluids. In Figure 20 the final configurations and pressure fields (at $t=1$ s) obtained by the two formulations are shown.

The graphs of Figure 19 and the pictures of Figure 20 show a very good agreement between the nodal and the elemental solutions for both cases, proving the feasibility of the proposed nodal approach also in the case of non-Newtonian problems. 
(a) $t=0.1 \mathrm{~s}$

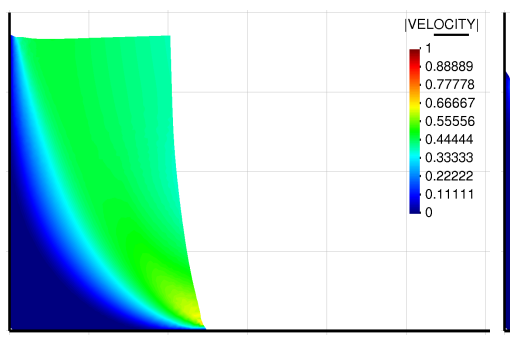

(d) $t=0.4 \mathrm{~s}$

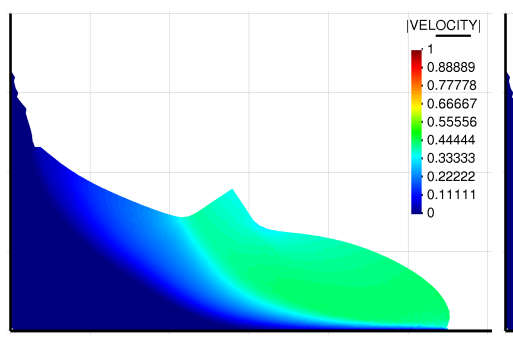

(b) $t=0.2 \mathrm{~s}$

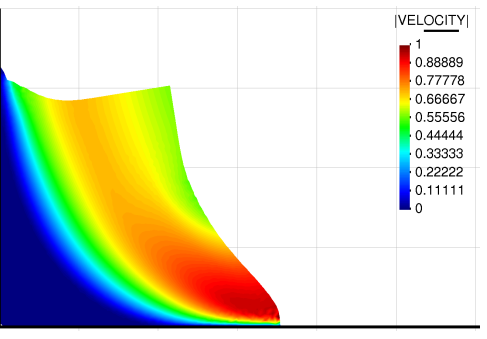

(e) $t=0.5 \mathrm{~s}$

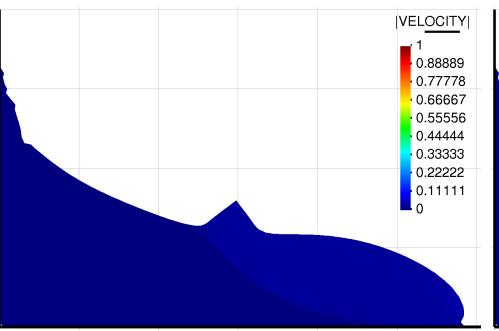

(f) $t=1.0 \mathrm{~s}$

(c) $t=0.3 \mathrm{~s}$
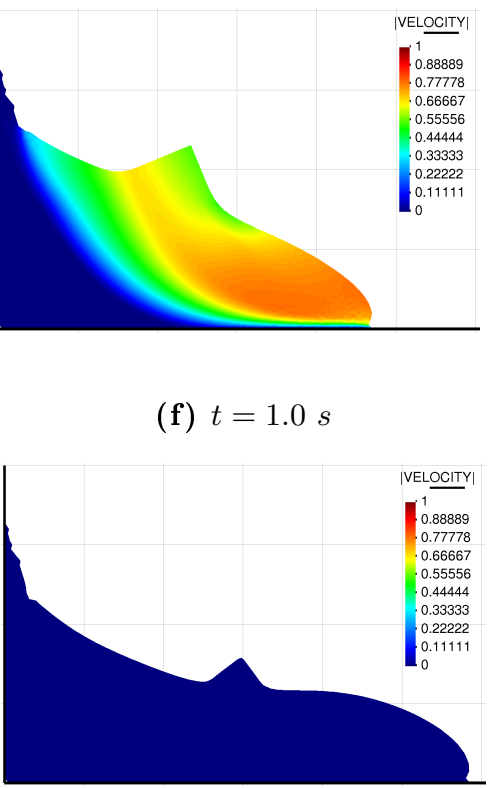

Figure 18: Dam break of a non-Newtonian fluid. Velocity field for the case $\tau_{0}=300 \mathrm{~Pa}$.

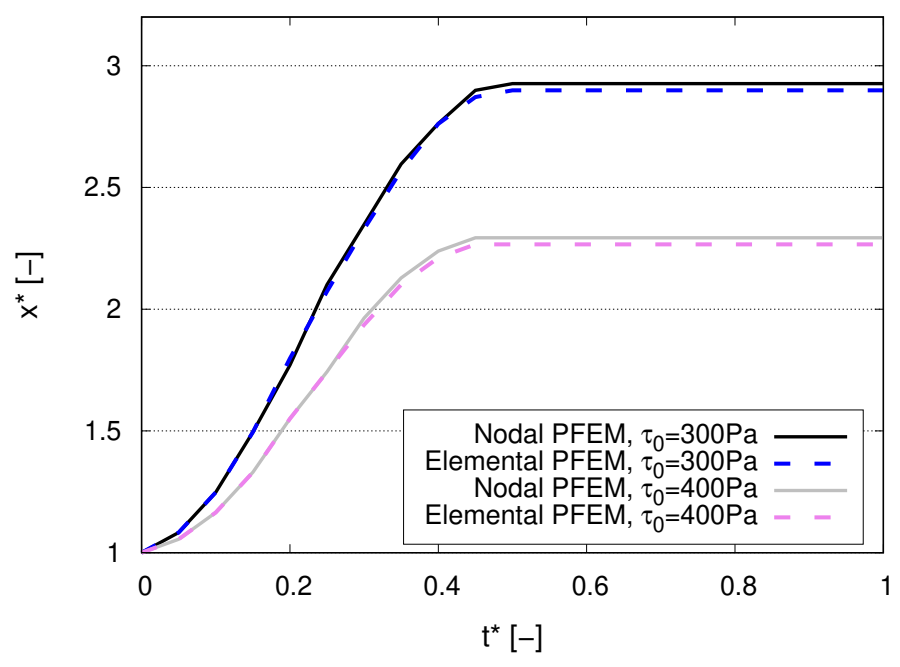

Figure 19: Dam break of a non-Newtonian validation. Dimensionless time evolution of the front wave position obtained with elemental [32] and nodal PFEM. 
(a) Elemental PFEM [32], $\tau_{0}=300 \mathrm{~Pa}$

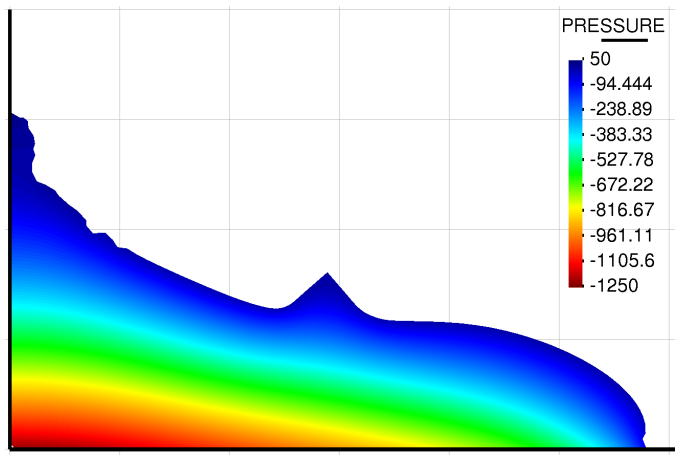

(c) Elemental PFEM [32], $\tau_{0}=400 \mathrm{~Pa}$

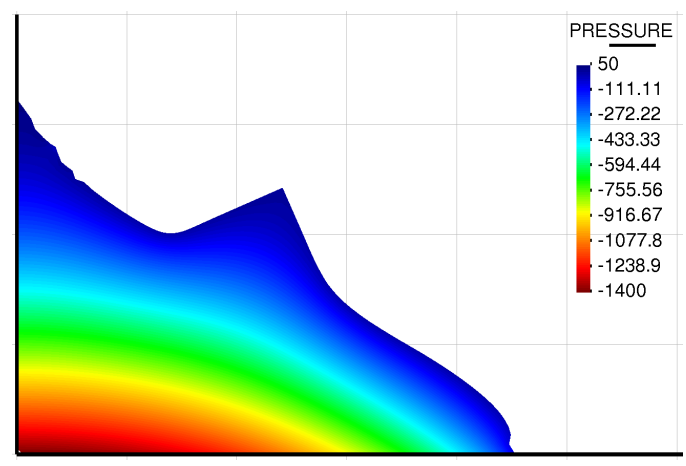

(b) Nodal PFEM, $\tau_{0}=300 P a$

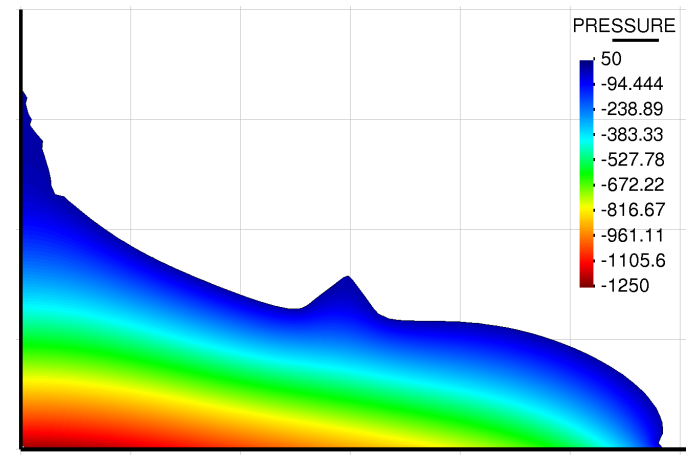

(d) Nodal PFEM, $\tau_{0}=400 \mathrm{~Pa}$

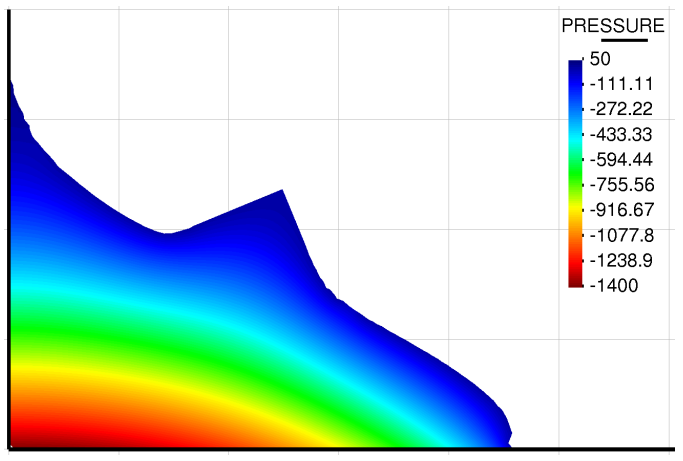

Figure 20: Dam break of a non-Newtonian fluid results. Pressure field at $t=1.0 \mathrm{~s}$ obtained by the proposed nodal PFEM and the elemental PFEM [32. Results for $\tau_{0}=300 P a$ and $\tau_{0}=400 P a$, in the first and second row, respectively. 


\section{Conclusions}

In this work, a novel PFEM approach with nodal integration has been presented and analyzed for free-surface fluid problems. In the proposed nodal PFEM, the governing equations are discretized over a FEM mesh, as in the standard PFEM, but their integration is not performed at the Gauss points, but rather at the nodes, where also stresses and strains are defined. This not only enables us to avoid convection of historical variables upon remeshing, but it also improves the convergence behavior of the numerical scheme, also when historical variables are not used, as e.g. in fluid dynamics.

The nodal PFEM has been validated against several 2D and 3D problems involving Newtonian and non-Newtonian fluids, and its performances have been compared with analogous PFEM with elemental integration. In all the analyzed tests, the nodal PFEM has shown to be able to reproduce accurately the available analytical solutions and the numerical and experimental results of the literature. It has also been shown that the nodal PFEM can be used for demanding 3D problems.

In terms of accuracy, the results of the nodal formulation have mirrored with high fidelity the elemental ones. This has also been shown in non-Newtonian fluids problems, which are particularly critical due to the dependency of the fluid viscosity on the strain measures.

Concerning mesh convergence, the nodal and elemental PFEMs have shown to have the same quadratic convergence rate for velocities and pressure, although the elemental formulation gave a slightly reduced error for the same mesh. On the other hand, the nodal PFEM has shown to guarantee a higher accuracy for the stresses and an almost quadratic convergence rate, whereas the convergence is only linear for the elemental PFEM. The convergence test has also shown that the elemental and nodal formulations have a different convergence path to the exact solution. In a

nodal approach, the convergence is from the stiffness side (the coarser is the mesh, the less stiff is the response) while in an elemental approach it is from the compliance side.

It has also been shown that the nodal approach is less affected by element distortion than the elemental method. This is of great importance in a PFEM framework, as it allows us to reduce the remeshing events and their associated drawbacks.

In terms of non-linear convergence and mass conservation properties, nodal and elemental PFEM have shown similar behavior, although a slightly faster convergence has been observed in the nodal PFEM solution.

A quantitative comparison of computational time between nodal and elemental PFEMs has not 
been performed in this work. This is because the proposed nodal solver has been implemented into a numerical platform optimized for standard elemental FEM, and thus the comparison would be not fair.

However, we can extrapolate some considerations. Considering that we are using an implicit time integration scheme with a fixed time step size, the two methods used the same number of time steps. Consequently, having proved that the nodal approach converges faster on average than the elemental one (see Figure 11), it follows that globally the nodal method required a smaller number of total non-linear iterations than the elemental approach. Furthermore, we showed that the nodal solution is less sensitive to element distortion (as shown e.g. in Figure 8), and consequently, it allows for a less frequent remeshing leading to a further saving of computational time. On the other hand, the operations of assembly are expected to be more expensive in a nodal approach than in an elemental one, due to the larger support of each node and the consequent larger bandwidth of the stiffness matrix, and, for the same mesh, the linear system solution is more computationally expensive. In summary, from the one hand, the nodal solver requires fewer iterations and it is less prone to mesh distortion, from the other, each iteration will be more expensive than the elemental one. A detailed analysis of the performances in terms of CPU time would be the subject of interesting future work.

The results obtained have demonstrated the potential of the PFEM with nodal integration for free-surface fluid dynamics problems. After the recent application and validation of a nodal PFEM for solid mechanics problems [10, 9], this work constitutes a further step towards a unified nodal Lagrangian framework for large deformation processes, also in the presence of liquid-solid transition, where the storage of internal variables is unavoidable.

\section{Acknowledgments}

The first author, beneficiary of an AXA Research Fund post-doctoral grant (grant number 2017-AXA-PDOC1-099), acknowledges AXA for its economic support. The Spanish Ministry of Economy and Competitiveness (Ministerio de Economia y Competitividad, MINECO) through the project PRECISE (BIA2017-83805-R) is also greatly acknowledged. 


\section{References}

[1] W. Zheng and G.R. Liu, Smoothed Finite Element Methods (S-FEM): An Overview and Recent Developments, Archives of Computational Methods in Engineering, 25:397-435 (2018)

[2] Z.Q. Zhang and G.R. Liu and B.C. Khoo, Immersed Smoothed finite element method for two dimensional fluid-structure interaction problems, International Journal of Numerical Methods in Engineering, 90, 1292-1320 (2012)

[3] E. Li and Z. Zhang and C.C. Chang and G.R. Liu and Q. Li, Numerical Homogenization for incompressible materials using selective smoothed finite element method, Composite Structures, $123,216-232(2015)$

[4] G.R. Liu and G.Y. Zhang, Upper bound solution to elasticity problems: A unique property of linearly conforming point interpolation method (LC-PIM), International Journal of Numerical Methods in Engineering, 74, 1128-1161 (2008)

[5] G.R. Liu and T.T. Nguyen and K.Y. Dai and K.Y. Lam, Theoretical aspects of the smoothed finite element method (SFEM). International Journal of Numerical Methods in Engineering, 71(8), 902-930 (2006)

[6] G.R. Liu and T.T. Nguyen and H. Nguyen-Xuan and K.Y. Lam, A node-based smoothed finite element method (NS-FEM) for upper bound solutions to solid mechanics problems. Computers and Structures 87:14-26 (2009)

[7] T. Nguyen-Thoi and G.R. Liu and K.Y. Lam G.Y. Zhang, A face-based smoothed finite element method (FS-FEM) for 3D linear and geometrically non-linear solid mechanics problems using 4node tetrahedral elements. International Journal of Numerical Methods in Engineering, 78(3), $324-353$ (2009)

[8] W. Zeng and G.R. Liu and D. Li and X.W. Dong, A smoothing technique based beta finite element method ( $\beta$ FEM) for crystal plasticity modeling. Computers and Structures, 162, 48-67 (2016)

[9] W.H. Yuan and B. Wang and W. Zhang and Q. Jiang and X.T. Feng, Development of an explicit smoothed particle finite element method for geotechnical applications, Computers and Geotechnics, 106, 42-51 (2019) 
[10] W. Zhang, W. Yuan, B. Dai, Smoothed particle finite-element method for large-deformation problems in geomechanics, International Journal of Geomechanics, 18 (4), 04018010 (2018)

[11] C.R. Dohrmann and M.W. Heinstein and J. Jung and S.W. Key and W.R. Witkowski, Nodebased uniform strain elements for three-node triangular and four-node tetrahedral meshes, $47(9), 1549-1568$ (2000)

[12] J. Bonet and A. Burton, An averaged nodal deformation gradient linear tetrahedral element for large strain explicit dynamic applications. Communications in Numerical Methods in Engineering, 17, 551-561 (2001)

[13] L. Zhang and J. Ouyang and X.H. Zhang, On a two-level element-free Galerkin method for incompressible fluid flow, Applied Numerical Mathematics 59, 1894-1904 (2009)

[14] M. Philippou and Y. Damianou and X. Miscouridou and G.C. Georgiou, Cessation of Newtonian circular and plane Couette flows with wall slip and non-zero slip yield stress, Meccanica, $52,2081-2099(2017)$

[15] S.R. Idelsohn and E. Oñate and F. Del Pin, The particle finite element method: a powerful tool to solve incompressible flows with free-surfaces and breaking waves, International Journal for Numerical Methods in Engineering, 61, 964-989 (2004)

[16] E. Oñate and S.R. Idelsohn and F. Del Pin and R. Aubry, The particle finite element method. An overview, International Journal for Computational Methods, 1, 267-307 (2004)

[17] A. Larese and R. Rossi and E. Oñate and S.R. Idelsohn, Validation of the particle finite element method (PFEM) for simulation of free surface flows, International Journal for Computer-Aided Engineering and Software, 25, 385-425 (2008)

[18] S.R. Idelsohn and N. Calvo and E. Oñate, Polyhedrization of an arbitrary point set, Computer Methods in Applied Mechanics and Engineering, 92 (22-24), 2649-2668 (2003)

[19] P. Ryzhakov and E. Oñate and S.R. Idelsohn, Improving Mass Conservation in Simulation of Incompressible flows, International Journal of Numerical Methods in Engineering, 90, 1435$1451(2012)$ 
[20] A. Franci and E. Oñate and J.M. Carbonell, On the effect of the bulk tangent matrix in partitioned solution schemes for nearly incompressible fluids, International Journal for Numerical Methods in Engineering, 102 (3-4), 257-277 (2015)

[21] H. Edelsbrunner and E.P. Mucke, Three dimensional alpha shapes, ACM Trans Graphics, 13, 43-72 (1999)

[22] H. Edelsbrunner and T.S. Tan, An upper bound for conforming Delaunay triangulations, Discrete and Computational Geometry, 10 (2), 197-213 (1993)

[23] F. Brezzi, On The Existence, Uniqueness And Approximation Of Saddle-Point Problems Arising From Lagrange Multipliers, Revue française d'automatique, informatique, recherche opérationnelle. Série rouge. Analyse numérique, 8 (R-2), 129-151 (1974)

[24] E. Oñate and A. Franci and J.M. Carbonell, Lagrangian formulation for finite element analysis of quasi-incompressible fluids with reduced mass losses,International Journal for Numerical Methods in Fluids, 74 (10), 699-731 (2014)

[25] A. Franci and M. Cremonesi, On the effect of standard PFEM remeshing on volume conservation in free-surface fluid flow problems, Computational Particle Mechanics, 4 (3), 331-343 (2017)

[26] S. Koshizuka and Y. Oka, Moving-particle semi-implicit method for fragmentation of incompressible fluid, Philosophical Nuclear science and engineering, 123 (3), 421-434 (1996)

[27] S. Meduri and M. Cremonesi and U. Perego, An efficient runtime mesh smoothing technique for 3D explicit Lagrangian free-surface fluid flow simulations, International Journal for Numerical Methods in Engineering, 113, 43-64 (2018)

[28] S. Koshizuka and Y. Oka, Moving particle semi-implicit method: fully Lagrangian analysis of incompressible flows, Proceedings of the European Congress on Computational Methods in Applied Sciences and Engineering (ECCOMAS), Barcelona (Spain) (2000)

[29] A. Franci and I. de-Pouplana and G. Casas and M.A. Celigueta and J. González-Usua and E. Oñate, PFEM-DEM for particle-laden flows with free surface,Computational Particle Mechanics, https://doi.org/10.1007/s40571-019-00244-1 (2019) 
[30] A. Franci and E. Oñate and J.M. Carbonell and M. Chiumenti, PFEM formulation for thermocoupled FSI analysis. Application to nuclear core melt accident, Computer Methods in Applied Mechanics and Engineering, 325, 711-732 (2017)

[31] A. Franci and E. Oñate and J.M. Carbonell, Unified Lagrangian formulation for solid and fluid mechanics and FSI problems, Computer Methods in Applied Mechanics and Engineering, 298, $520-547$ (2016)

[32] A. Franci and X. Zhang, 3D numerical simulation of free-surface Bingham fluids interacting with structures using the PFEM, Journal of Non-Newtonian Fluid Mechanics, 259, 1-15 (2018)

[33] M.L. Cerquaglia and G. Deliége and R. Boman and V. Terrapon and J.P. Ponthot, Free-slip boundary conditions for simulating free-surface incompressible flows through the particle finite element method, International Journal for Numerical Methods in Engineering,110 (10), 921-946 (2016)

[34] S.R. Idelsohn and J. Marti and A. Limache and E. Oñate, Unified Lagrangian Formulation For Elastic Solids And Incompressible Fluids: Applications to Fluid-Structure Interaction Problems Via The PFEM, Computer Methods In Applied Mechanics And Engineering, 197, $1762-1776(2008)$

[35] X. Zhang and K. Krabbenhoft and D.M. Pedroso and A.V. Lyamin and D. Sheng and M. Vicente da Silva and D. Wang, Particle Finite element analysis of large deformation and granular flow problems, Computer and Geotechnics, 54, 133-142 (2013)

[36] A. Franci and M. Cremonesi, 3D regularized $\mu(\mathrm{I})$-rheology for granular flows simulation, Journal of Computational Physics, 378, 257-277 (2019)

[37] E. Oñate and R. Rossi and S. R. Idelsohn and K. Butler, Melting and spread of polymers in fire with the Particle Finite Element Method, International Journal of Numerical Methods in Engineering, 81 (8), 1046-1072 (2010)

[38] M. Cremonesi and A. Frangi and U. Perego, A Lagrangian finite element approach for the simulation of water-waves induced by landslides, Computer and Structures, 89, 1086-1093 (2011) 
[39] M.A. Puso and J.S. Chen and E. Zywicz and W. Elmer, Meshfree and finite element nodal integration methods, International Journal of Numerical Methods in Engineering, 74, 416-446 (2008)

[40] M.A. Puso and J.R. Solberg, A formulation and analysis of a stabilized nodally integrated tetrahedral, International Journal of Numerical Methods in Engineering, 67, 841-867 (2006)

[41] G. Scovazzi and B. Carnes and X. Zeng and S. Rossi, A simple, stable, and accurate linear tetrahedral finite element for transient, nearly, and fully incompressible solid dynamics: a dynamic variational multiscale approach, International Journal of Numerical Methods in Engineering, $106,799-839$ (2016)

[42] T.C. Papanastasiou, Flows of Materials with Yield. Journal of Rheology, 31, 385-404 (1987)

[43] T. Belytschko and W.K. Liu and B. Moran and K.I. Elkhodadry, Nonlinear Finite Elements For Continua And Structures, John Wiley \& Sons, New York (2014)

[44] P. Krysl and B. Zhu, Locking-free continuum displacement finite elements with nodal integration. International Journal of Numerical Methods in Engineering, 76: 1020-1043 (2008)

[45] G. Castellazzi and P. Krysl, Displacement-based finite elements with nodal integration for Reissner-Mindlin plates. International Journal of Numerical Methods in Engineering, 80, 135$162(2009)$

[46] E. Artioli and G. Castellazzi and P. Krysl, Assumed strain nodally integrated hexahedral finite element formulation for elastoplastic applications, International Journal of Numerical Methods in Engineering, 99, 844-866 (2014)

[47] J. Bonet and A.J. Burton, A simple average nodal pressure tetrahedral element for incompressible and nearly incompressible dynamic explicit applications. Commun. Numer. Meth. Engng., 14, 437-449 (1998)

[48] J. Chen and S. Yoon and C. Wu, Non-linear version of stabilized conforming nodal integration for Galerkin mesh-free methods. Int. J. Numer. Meth. Engng., 53: 2587-2615. (2002)

[49] W. Quak and A.H. van den Boogaard and D. González and E. Cueto, A comparative study on the performance of meshless approximations and their integration, Comput Mech 48: 121 (2011) 